NASA Technical Memorandum 100677

\title{
Observed Tidal Braking in the Earth/Moon/Sun System
}

\author{
(DASE-TM-100077) CESEEVED TIIAL ERAKING IN N89-12104 \\ ILE EARTH/BCCAISL SYSTEN (ASA) $81 \mathrm{P}$ \\ CSCL $03 \mathrm{~B}$

$\begin{array}{ll}\text { Unclas } \\ 63 / 42 & 0171181\end{array}$

D. C. Christodoulidis, D. E. Smith,

R. G. Williamson, and S. M. Klosko 

NASA Technical Memorandum 100677

\section{Observed Tidal Braking in the Earth/Moon/Sun System}

D.C. Christodoulidis and D. E. Smith

Laboratory for Terrestrial Physics

Geodynamics Branch

Goddard Space Flight Center

Greenbelt, Maryland 20771

and

R. G. Williamson and S. M. Klosko

EGEG Wasbington Analytical Services Center, Inc.

Lanbam, Maryland 20706

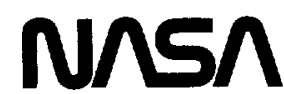

Natıonal Aeronautics and

Space Administration

Goddard Space Flight Center

Greenbelt. Maryland 20771 



\section{PREFACE}

The low degree and order terms in the spherical harmonic model of the tidal potential have been observed through the perturbations which are induced on near-earth satellite orbital motions. This recovery, which is the most complete dynamic model ever obtained, has been achieved through evaluating tracking observations on 17 different, mostly laser, satellites. A new improved GEM-T1 geopotential model, complete to degree and order 36, was estimated simultaneously with the 66 adjusted tidal coefficients. The gravitational and tidal models were developed using the 32000 Reference system with the adopted nutations of Wahr and the precession model of Lieske. The tidal recovery was made in the presence of an extended oceanographic model containing over 600 long wavelength coefficients from 32 major and minor tides. Since solid earth tides have identical perturbing frequencies as the ocean tides, the solid earth tidal model of Wahr was used as a basis for the recovery of the ocean tidal terms. This provided a complete description of the combined tidal potential sensed by these well tracked satellites. This tidal model (for all 32 adjusted and unadjusted tides) has then been used to calculate the secular change in the moon's mean motion due to tidal dissipation and the tidal braking of the earth's rotation. The secular change in the moon's mean motion due to tidal dissipation is found to be $-25.27 \pm 0.61$ arcsec $c y^{-2}$. Our estimate of the lunar acceleration agrees well with that observed from Lunar Laser Ranging techniques, which most recently found $-24.9 \pm 1.2 \operatorname{arcsec~cy}^{-2}$ (Newhall et al, 1986). The corresponding tidal braking of the earth's rotation is $-5.98 \pm .22 \times 10^{-22} \mathrm{rad} \mathrm{sec}^{-2}$. If the non-tidal braking of the earth due to the observed secular change in the earth's second zonal harmonic (Yoder et al, 1983) is considered, modern satellite techniques yield a total value of the secular change in the earth's rotation rate of -4.69 $\pm 0.36 \times 10^{-22}$ rad $\mathrm{sec}^{-2}$. 



\section{TABLE OF CONTENTS}

Preface

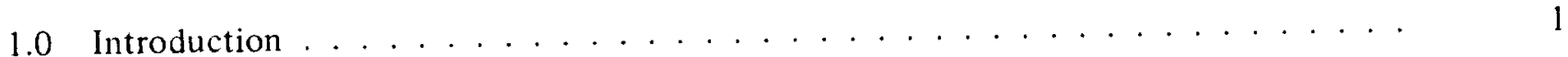

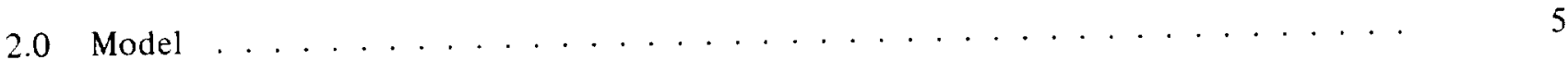

2.1 Apriori Solid Earth and Ocean Tide Models . . . . . . . . . . . 6

2.2 The GEM-Tl Solution Design ................... 9

3.0 The GEM-T1 Ocean Tidal Solution .................... 13

4.0 Secular Perturbations in the Moon/Sun Orbit . . . . . . . . . . . 17

5.0 Tidal Braking of the Earth's Rotation ................... 21

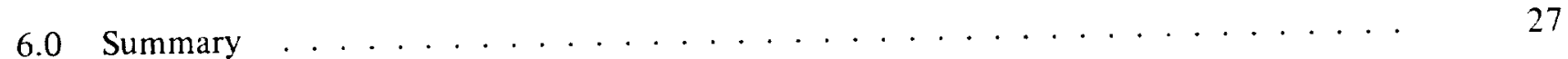

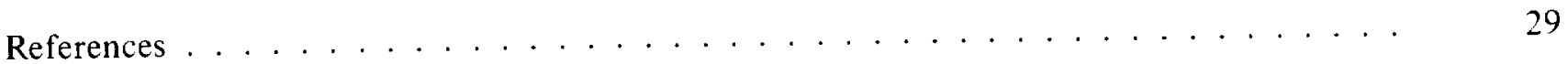

Appendix $\mathrm{A} \ldots \ldots \ldots \ldots \ldots \ldots \ldots \ldots \ldots \ldots \ldots$ A $\ldots \ldots \ldots \ldots \ldots$

Appendix B .............................. B 

The gravitational attraction of the sun and moon deform the earth, its oceans and its atmosphere. These deformations, known as tides, provide a rich spectrum of effects. Among those which are readily observable, the tides cause temporal variations in the external gravitational attraction the earth exerts on near-by orbiting objects and cause complex changes in the rate of the earth's rotation.

Any imperfect tidal response of the earth results in a tidal bulge which is not exactly aligned in the direction to the disturbing third body. This phase lag in the tidal response produces a torque which causes a transfer of angular momentum in the earth/moon/sun system. Since the ocean tides usually have much larger phase lags than the solid earth tides, they play a dominating role in this exchange of angular momentum. This transfer of angular momentum manifests itself as secular changes in the orbital elements of the disturbing bodies and in the braking of the earth's rotation rate.

The subject of tidal dissipation is a classical problem which is discussed at length in Lambeck (1980). Tidal dissipation has been a subject of intense interest in the geophysical, astronomical and oceanographical communities, because the redistribution of the earth's mass due to the tides is the recognized explanation for the observed acceleration in the earth's rotation and acceleration of the lunar orbit.

The earth/moon and earth/near-earth satellite systems have strong physical similarites. Man-made earth orbiting satellites have certain observational advantages over the moon. They have better known, and in most cases, laboratory measured physical characteristics. Man-made orbiting objects are generally easier to observe with great precision by earth-based ranging systems. The diversity of objects available for tracking also helps in the separation of contending forcing effects. 
Therefore, precise measurements to artificial satellites can play an important role in understanding the evolution of the earth/moon/sun system.

One disadvantage of resolving the tides from their effects on artificial satellites lies in the short observation history avallable on these orbits and the resulting inherent difficulty of separating secular from long periodic effects. However, the near earth satellite record is lengthening in time, permitting more robust tidal solutions. Centimeter level laser systems now routinely perform both lunar and near-earth satellite tracking. Direct comparisons and inferences can be made of the common satellite responses--elther that of the moon or an artificial satellite--to the external potential of the earth, especially those resulting from tidal forces.

Theoretical results associated with the evolution of the earth/ moon system have not previously been in close agreement with those obtained observationally. This disagreement has caused some to introduce other physical mechanisms, such as a temporal change in the gravitational constant, to bring theory and observation into balance (Van Flandern, 1975). Recoveries of tidal parameters from artificial satellites give important new insight into the earth's tidal response and the role these deformations play in the evolution of the lunar orbit.

The early work of Kaula (1964, 1969) first addressed the effects of solid earth tides on near-earth satellite orbits. Lambeck et al (1974) complicated the picture by showing the importance of ocean tides. Additionally, Lambeck (1977, 1980) has discussed a wide range of geophysical and astronomical consequences of these tides, such as their importance in the evolution of the earth/moon system, and braking the rotation of the earth. The purpose of this paper is to evaluate, in these contexts, the consequences of a new satellite based solution of earth and ocean tide parameters. 
Most of the early analyses for the recovery of tidal parameters from orbital perturbations were investigations of the long period tidal effects evidenced in the evolution of the orbit's mean Kepler elements. In most cases, the inclination and ascending node of the orbit were analyzed. These two out-of-plane components of the orbit have strong tidal signals, and these components are less sensitive to errors in other force models, such as the non-conservative effects. Tidal values from satellite analyses have been appearing in the literature since the late 1960's. Some of the major satellites used in our solution were previously analyzed in this manner; Douglas et al (1974) and Felsentreger et al (1976) studied Geos-1 and Geos-2, Cazenave and Daillet (1981) performed an analysis of Starlette, Goad and Douglas (1977) studied an Oscar Navy navigation satellite and Goad (1977) evaluated Geos-3.

The introduction of very precise laser ranging systems and an accompanying strong global tracking network made the combined recovery of tidal and geopotential terms possible from a direct analysis of satellite tracking data. As will be shown, the tides and the geopotential are closely related so that their effects can both be represented in the usual spherical harmonic form (Lambeck, 1980). Herein, the gravitational model is treated as the constant part of a time varying potential, with time dependent effects solved for at tidal frequencies. Williamson and Marsh (1985) broke important new ground in this direction with their tidal solution from starlette and Christodoulidis et al (1986) followed with a comparable analysis of Lageos.

In this paper we report on a new dynamical tidal solution which has been obtained at NASA/Goddard Space Flight center as part of an effort to develop an improved geopotential model (Marsh et al, 1987) in preparation for the 1991 launch of Topex/Poseidon (Born et al, 1986). In order to yield an accurate time-invariant gravity model, a complete separation of gravitational and tidal potential is necessary. This was 
accomplished through improved modeling, including the adoption of special tidal models which were more complete than those ordinarily available from oceanographic sources and the simultaneous least squares estimation of numerous geopotential and tidal coefficients. 
2.0 MODEL

A natural form for expressing the gravitational field for orbital calculations is in terms of spherical harmonic coeficients,

$V=\frac{G M}{r}\left\{1+\sum_{\ell=2}^{\infty} \sum_{m=0}^{\ell}\left(\frac{a_{e}}{r}\right)^{\ell} P_{\ell m}(\sin \phi)\left[c_{\ell m} \cos m \lambda+s_{\ell m} \sin m \lambda\right]\right\}$

where $G M$ is the earth's gravitional constant (including the atmosphere), $a_{e}$ is the earth's mean equatorial radius, $P_{l m}$ is the associated Legendre function of degree $\ell$ and order $m$, and $r, \phi$ and $\lambda$ are the distance from the center of mass, latitude and longitude (e.g. Kaula, 1966). A number of the potential coefficients $C_{\ell m}$ and $S_{\ell m}$ are considered to be time invariant unknowns and are allowed to adjust based upon observed orbital motion.

It is shown (Appendix 1) that in a system where the disturbing bodies (sun and moon) are referred to the ecliptic, and the perturbed body (the artificial satellite) is referred to the equator, the second degree tide potential is given by:

$$
v_{S E}=\sum_{f} k_{2, f} \bar{A}_{f} G_{D}\left(\frac{3-m}{3}\right)\left(\frac{R}{r}\right)^{3} P_{2 m}(\sin \phi) \cos \alpha_{f}
$$

for the solid earth and by

$$
v_{\text {ocean }}=\sum_{f} \sum_{\ell, q, \pm} 4 \pi \mathrm{GR} p_{0}\left(\frac{1+k_{\ell}^{\prime}}{2 \ell+1}\right) C_{\ell q, f}^{ \pm}\left(\frac{R}{r}\right)^{\ell+1} P_{\ell q}(\sin \phi) \cos \alpha_{\ell q, f}^{ \pm}
$$


for the oceans. This spherical harmonic form provides a linear response at each constituent $f$ in the tide-generating potential. The tidal and gravitational potentials combine to perturb the orbit. As can be seen in the above equations, the second degree tidal potential is equivalent to that due to gravitation with tidally driven, temporally varying second degree harmonics. Aliasing of the gravitational solution can result if there is a poor temporal sampling of these tidal effects and/or if the tides are poorly modeled. The principal earth and ocean tides, their Doodson numbers and appropriate Darwinian correspondancies are shown in Table 1 .

The principal perturbations due to solid earth and ocean tides have identical frequencies on satellite orbits. Consequently, earth and ocean tidal parameters cannot be recovered separately from the analysis of near-earth tracking data. In our solution, an adjustment is made to a set of ocean tidal parameters, which corrects the observed temporal exterior potential of the earth relative to an adopted solid earth tide model and relative to a partially unadjusted base ocean tidal model. By design within the solution, a sufficient number of independent tidal terms have been adjusted at their dominant frequencies. This solution and its interpretation form the basis of this paper.

\subsection{A PRIORI SOLID EARTH AND OCEAN TIDE MODELS}

For the solid earth tides, the frequency dependent model developed by Wahr (1979) has been adopted and held unadjusted within the solution (Table 2). All of the remaining solid earth tides are modeled with a frequency independent Love number of $k_{2}=.30$ with zero phase. Their tidal potential is evaluated in the time domain from lunar and solar ephemerides. 
Schwiderski (e.g., 1983, 1981, 1980) and Parke (1982) have analyzed global data acquired by tidal stations and have estimated oceanographic models for the major ocean tides. These models have been spectrally decomposed using quadrature under the assumption that the tidal amplitude $(\Delta)$ and phases $(\psi)$ are known at all points on the globe (where $\Delta \equiv 0$ for land areas). The $\Delta_{f} \cos \psi_{f}$ and $\Delta_{f} \sin \psi_{f}$ are computed on a uniform dense grid of points on the earth's surface to solve for the $a_{\ell m, f}, b_{\ell m, f}, c_{\ell m, f}$ and $d_{\ell m, f}$ used to represent each tide in harmonic form:

$$
\begin{aligned}
& \Delta_{f} \cos \psi_{f}=\sum_{\ell=0}^{\infty} \sum_{m=0}^{\ell}\left(a_{\ell m, f} \cos m \lambda+b_{\ell m, f} \sin m \lambda\right) P_{\ell m}(\sin \phi) \\
& \Delta_{f} \sin \psi_{f}=\sum_{\ell=0}^{\infty} \sum_{m=0}^{\ell}\left(c_{\ell m, f} \cos m \lambda+d_{\ell m, f} \sin m \lambda\right) P_{\ell m}(\sin \phi)
\end{aligned}
$$

For each tide constituent ( $f$ ), the values used for orbit computations are obtained as:

$$
\begin{aligned}
& c_{\ell m, f}^{+} \cos \zeta_{\ell m, f}^{+}=\frac{1}{2}\left[a_{\ell m, f}-d_{\ell m, f}\right] \\
& c_{\ell m, f}^{+} \sin \zeta_{\ell m, f}^{+}=\frac{1}{2}\left[b_{\ell m, f}+c_{\ell m, f}\right] \\
& c_{\ell m, f}^{-} \cos \zeta_{\ell m, f}^{-} \quad \frac{1}{2}-\left[a_{\ell m, f}-d_{\ell m, f}\right] \\
& c_{\ell m, f}^{-} \sin \zeta_{\ell m, f}^{-} \frac{1}{2}-\left[c_{\ell m, f}+b_{\ell m, f}\right]
\end{aligned}
$$


The expansion is taken out to some maximum order, lmax. The ocean tidal phases in the oceanographic models can be related to that of eq. 3 by :

$$
\varepsilon_{f}=\zeta_{f}-\frac{\pi}{2}+\pi \delta_{o n}+x
$$

where $\delta_{\text {om }}$ is the Kronecker delta (where $m=0$ denotes the long period, $m=1$ the diurnal and $m=2$ the semi-diurnal tides) and

$$
x=\mid \begin{array}{ll}
0 & \text { positive Doodson coefficients } \\
\pi & \text { negative Doodson coefficients }
\end{array}
$$

Note that for our major tides, $x=\pi$ only for $K 1, \varepsilon_{\mathrm{f}}$ is used in this form in Appendix 1.

There are many minor ocean tides which are not developed directly from oceanographic data due to the computational burden required for precise solutions of the Laplace Tidal Equations. Because of the coupling of the satellite motion and the disturbing body, many of these tides have significant effects on satellite orbits and are a source of allasing in the recovery of satellite derived ocean tidal models if not properly accounted for. This is especially true of the numerous sideband constituents which typically have an amplitude of $10 \%$ of the major tide at a nearby frequency. Total neglect of these sideband tides significantly degrades the accuracy of the adjustment for the terms defining the major tides. To calculate models for many minor ocean tides which were otherwise unavailable, an algorithm was developed and tested. Figure 1 diagrams the predictive method which was developed for the computation of any tide constituent and its errors given its frequency. The algorithm uses linear admittances to predict any tide in the long period, semi-diurnal and diurnal bands (Christodoulidis et al, 1986a). 
A sensitivity analysis was performed using the linear orbit perturbation theory of Appendix 1 to isolate those tides which were important for the satellite solution, and ocean-based tidal models were developed for those found to be satellite sensitive. The long wavelength coefficients of the ocean tides were adjusted simultaneously with the geopotential if sufficient sensitivity existed within the satellite observations. Otherwise, the ocean tidal terms were held fixed at their oceanographically determined values. Note that a change in the solid earth model is accomodated by a comparable change in the solved for ocean tides of the same frequency. This is further discussed in Section 3. Table 3 summarizes the complete set of ocean tidal terms which were modeled in the GEM-T1 solution and indicates which terms (if any) were adjusted within each tidal frequency.

Due to attenuation with altitude, most near-earth satellites are insensitive to ocean tidal terms above degree 6 and are most sensitive to the long period perturbations which arise exclusively from the prograde terms in the harmonic expansion. R. Eanes of the University of Texas has shown (private communications) that there are a few ocean tidal terms which we have neglected. These neglected terms are not of the same order as the dominant terms and resemble m-daily gravity effects of a few $\mathrm{cm}$ amplitude in orbital perturbations. As m-daily effects, these neglected tidal terms can cause an aliasing error in the geopotential recovery, but they are quite distinct from the tidal parameters adjusted herein.

\subsection{THE GEM-T1 SOLUTION DESIGN}

The recent GEM-T1 solution (Marsh et al., 1987, in press) using significantly more and better tracking data was a complete re-iteration of Goddard Space Flight Center's general gravitational model solutions. The availability of a super computer (the Cyber 205) and the 
vectorization of our major software tools greatly impacted the timeframe required to complete this work. GEM-T1 was carefully designed to be a general gravitational field (i.e., not a "tailored field" for any specific orbit). GEM-T1 was solved in the J2000 Reference System (with IAU 1980/Wahr nutation and modern reference constants being adopted). This reference system was generally based on the Merit Standards (Melbourne, et al., 1983). A summary of the reference parameters adopted within GEM-T1 can be found in Table 4.

The GEM-T1 solution made use of a large subset of the precise third generation laser tracking data (with tracking precision better than $5 \mathrm{~cm}$ ) taken on 7 geodetic satellites. Strong Tranet Doppler data sets from Seasat and Oscar 14 were also used. Interestingly, each of the geodetic satellites which have been studied earlier for tides have now been reanalyzed as part of this simultaneous multi-satellite gravitational and tidal solution. In each case, improved tracking data sets (i.e., better precision and global distribution) have been utilized in the formation of GEM-T1. Furthermore, a simultaneous adjustment of tidal and geopotential terms has yielded more complete and accurate dynamic tidal results. The GEM-T1 gravitational model is complete in spherical harmonics to degree and order $36 ; 66$ tidal parameters representing the longest wavelength coefficients of 12 major tides were simultaneously adjusted in the solution. Approximately 550 other ocean tidal terms were used to model long wavelength ocean tidal variability for 32 major and minor tides.

In all, 17 satellites were included in the GEM-T1 solution. of the 17, weaker data sets from six low inclination objects were used specifically to help resolve the zonal harmonics of the field. The strong data sets were those acquired by recent laser and Doppler systems. Table 6 and Figure 2 describe the orbital characteristics of the satellites used in the formation of GEM-T1. 
The summary of the data in Table 5 reveals that precise laser tracking played a dominant role in defining the GEM-T1 geopotential and tidal models. of particular importance, two laser satellites, Lageos and Starlette, made strong contributions to both the tidal and geopotential field recoveries. These are completely passive orbiting objects which were exclusively designed to serve as space-based laser targets. Both satellites are extremely dense spheres (area to mass ratios of .00069 and $.00096 \mathrm{~m}^{2} \mathrm{~kg}^{-1}$ respectively), covered by 1 aser corner cubes, and are in orbits designed to minimize non-conservative forcing effects. Lageos orbits at nearly an earth radius above the earth and senses only the longest wavelength gravitational and tidal fields. Starlette, orbiting at a much lower altitude of $-1000 \mathrm{~km}$, experiences a rich spectrum of tidal and gravitational perturbations and is highly complementary to Lageos for the separation of long and short wavelength gravitational and tidal terms. Figures 3.1 and 3.2 show the complimentary tidal perturbation spectra sensed in the inclination and ascending node perturbations of starlette and Lageos. Both of these satellites are tracked on a high priority basis by a global network of laser tracking stations and have extensive observation sets which have been acquired under NASA's Crustal Dynamics Project activities (Coates et al, 1985), Project MERIT, and the WEGENER Campaign.

A summary of the principal periods of the solid earth and ocean tides on the major satellites utilized in the solution can be found in Figure 4. These tides perturb the satellite orbits with dominating long period effects. On the earth's surface, the largest tides are found in either the diurnal or semi-diurnal band. The precession of a satellite's ascending node and not the earth's rotation is responsible for the wide range of these satellite periodicities. 
$-1$ 


\subsection{THE GEM-T1 OCEAN TIDAL SOLUTION}

Table 3 has summarized the ocean tidal terms which were selected for adjustment in the GEM-T1 solution (that is, adjusted simultaneously with the gravitational field). Tables 7.1 through 7.4 present the recovered ocean tidal spherical harmonic coefficients respectively for each degree. The values shown for the coefficient and phase uncertainties were taken from a covariance matrix which was calibrated for the geopotential coefficient uncertainties (see Marsh et al, 1986). We believe this calibration also applies for the tides. This calibration gave realistic (one sigma) uncertainties for the estimated tidal and geopotential terms. Because the ocean tidal terms are inseparable from those of the solid earth at the same frequencies, the uncertainty specified for a given tidal term does not reflect changes which would arise from modifications to the fixed solid earth tides which are modeled. Rather, these uncertainties represent the error in the sum of the solid earth and ocean tidal models at a given frequency mapped into the spherical harmonic decomposition of the ocean tide. These uncertainties represent commission errors (in a strict sense) in modeling the external potential of the earth at tidal frequencies when using the complete ocean tidal description available in GEM-T1 plus the Wahr solid earth tidal models. Neglect of an adjustment for the very long period tides (e.g. the 18 year tide) is a potential source of aliasing which is not considered in the error estimates which are presented.

Note that the GEM-T1 recovered tide coefficients do not require correction for sideband effects as was necessary in Cazenave \& Daillet (1981) and in Williamson and Marsh (1985). The errors assoclated with the sideband constituents have been minimized because the sideband terms are explicitly modeled in our solution and the satellite data spans a significant number of years. 
In the current solutions, the Wahr Earth Model is adopted to describe the solld earth's tidal response. This model was developed assuming a uniformly rotating earth comprised of an elastic inner core and mantle and a liquid core. Dehant $(1986 a, b, c)$ has estimated the effects of anelasticy on different components of the earth's tidal response using models developed by Zschau (1985) and others. She has shown that the neglect of anelasticity may be the cause of some of the discrepancy in the observed versus predicted gravimetric factors at tidal frequencies. Wahr and Bergen (in press) have also addressed the question of the effect of anelasticity on the earth tides (and in the tidally induced variation in the earth's rotation rate) and have estimated upper and lower bounds for these effects. They have noted that observational data of sufficient precision is lacking to permit a meaningful estimate of anelastic parameters directly, even though recent recoveries of nutation amplitudes by space based technologies like VLBI put us on the threshold of doing so. Wahr and Bergen (ibid) show that for the diurnal and semi-diurnal tidal frequencies, the lower and upper bound for the anelastic effect on $k_{f}$ is typically .002 and .015 respectively. These assessments agree well with those developed by Dehant. Using the midpoint of these upper and lower bounds shown by Wahr and Bergen for the anelastic contribution at specific tidal frequencies and a $1 \%$ change in $k_{2}$ for all other tides in our model, we have performed a sensitivity study by re-estimating all 66 ocean tide coefficients in the presence of this changed solid earth tide model. The adjusted 2nd degree ocean tidal coefficients which result generally change in phase by approximately 3 degrees and in amplitude hy less than 10\%. However, the resulting external potential described by the complete ocean and solid earth model remains nearly constant (within one third of the standard deviations given). This test of the stability of the model's external potential compared the calculated values of the lunar acceleration and the braking of the earth's rotation with the values obtained with the original wahr model. 
The GEM-T1 satellite determined values are also compared to those estimated from the oeanographic tidal solutions of Schwiderski and Parke (Tables 7.1 to 7.4). Generally, the comparison of satellite and oceanographic tidal values is quite good. The variation seen between the two oceanographic tidal solutions is often larger than the uncertainty of the dynamic satellite solution. We believe these results confirm that the adopted wahr solid earth tidal model is quite reasonable and that the solution for long wavelength ocean tidal terms is realistic when compared to those observed oceanographically. These satellite determined ocean tidal coefficients can provide effective constraints within future oceanographic-based model developments.

of special interest are the second degree values for the $M_{2}$ tide where the amplitudes show large disagreement between oceanographic models. For this term, which dominates the lunar evolution, the GEM-T1 satellite result favors the value obtained by Parke. For the $o_{1}$ second degree values, a large disagreement is also present, only this time the GEM-T1 satellite results favor the Schwiderski model.

The 1 arge uncertainty in the oceanographic models (as evidenced by the level of disagreement they exhibit) prevents an accurate separation of solid earth and ocean tidal contributions in the observed total effects seen by orbiting objects. As explained in Williamson and Marsh (1985), significant improvements are also required in the modeling of atmospheric tides, atmospheric pressure loading over both the oceans and the continents, the radiational potential, and other such subtle effects. This lack of strong earth-based modeling is the reason that satellite tidal analyses cannot yet be expected to contribute significantly to assessing tidal dissipation in the solid earth. Zschau (1986) comes to the same compelling conclusion in his attempt to interpret the $M_{2}$ solid tide dissipation from combined satellite-ocean tide solutions. 
Table 8 compares 2nd and 4 th degree ocean tidal coefficients obtained by other satellite studies with the values from the GEM-T1 solution. Our satellite values seem to be consistent with most previously reported satellite results, especially the $M_{2}$ analyses. For $M_{2}$, both the second and fourth degree terms are in good agreement. Note the Cazenave and Daillet (1981) values for $0_{1}$ shown were taken from Williamson and Marsh (1985), who corrected them to refer to the Wahr nutations from the original values using the woolard nutations.

The GEM-T1 ocean tidal model is a significant improvement over earlier studies. It alone is based upon the simultaneous adjustment of geopotential and tidal terms, it uses the wahr nutations, it has modeled 550 presently unrecoverable sideband and higher degree ocean tidal terms, and has been derived from complete tracking information on a multiplicity of satellites. Most, if not all, of these factors are significant limitations in the earlier satellite ocean tidal investigations. Because of the nature of present model improvements, the systematic errors in the earlier investigations are probably comparable to or larger than any error estimates found in previous analyses. We have therefore not tabulated the comparitive error estimates from these earlier investigations. 
4.0 SECULAR PERTURBATIONS IN THE MOON/SUN ORBIT

The observed secular acceleration in the moon's orbit is dominated by tidal dissipation within the earth's oceans. The secular variation of the Kepler elements of the moon and apparent sun are calculated using the tidal potential in the Lagrange Planetary Equations, evaluating those terms of zero frequency as detalled in Appendix 2. The ecliptic Kepler rates take the form:

$$
\begin{aligned}
& \frac{\mathrm{da}^{*}}{d t}=K_{a}^{*} \quad C_{2 m, f}^{+} \sin \varepsilon_{2 m, f}^{+} \\
& \frac{d e^{*}}{d t}=K_{e}^{*} \quad C_{2 m, f}^{+} \sin \varepsilon_{2 m, f}^{+} \\
& \frac{d i^{*}}{d t}=K_{1}^{*} \quad C_{2 m, f}^{+} \sin \varepsilon_{2 m, f}^{+}
\end{aligned}
$$

The * stands for either the moon or the sun (there is also a small change in the apparent solar orbit); $m=0$ for long period tides (LP), $m=1$ for diurnal tides (D) and $m=2$ for semi-diurnal tides (SD). These labels are used in summary tables. $C_{2 m}^{+}$and $\varepsilon_{2 m}^{+}$are the second degree tidal amplitudes and phases obtained from the GEM-T1 tidal solution or the background unadjusted tidal models, and $K_{a}^{*}, K_{e}^{*}$, and $K_{i}^{*}$ are calculated from analytical theory as shown in Appendix 2.

To obtain the third body accelerations from the GEM-T1 tidal model, it is necessary to consider the contributions of all of the adjusted and unadjusted second degree terms. The Wahr solid earth tidal model has no anelasticity and thus there is no phase lag in the earth's lunisolar tidal response and no resulting torque on the lunar orbit. The complete second degree ocean tide model includes the unadjusted tidal coefficients derived from admittances (Table 9) which have been included in all of our near-earth orbital calculations as well as those terms adjusted from the satellite data (as given in Table 7.1). 
The secular changes in the Kepler elements of the moon and apparent solar orbit based on the GEM-T1 model due to tidal energy dissipation in the earth's oceans are summarized in Table 10. The effect of the earth's ocean tides on the lunar orbit is reasonably well determined and can be compared with lunar evolution observations. However, only the change in the apparent solar semi-major axis is measured significantly in the sun/earth case. The uncertainties in the Kepler-element rates which are given have been obtained from a formal RSS propagation of the tidal coefficent errors (as given in Table 7.1) combined with a $10 \%$ estimated uncertainty for all unadjusted terms shown in Table 9.

Using the simple relationship derived from Kepler's Law, the secular perturbation of the lunar mean motion is obtained from the semimajor axis rate as

$$
\frac{\mathrm{dn}}{\mathrm{dt}}=\frac{-3}{2} \frac{\mathrm{n}^{*}}{\mathrm{a}^{*}} \frac{\mathrm{da}}{\mathrm{dt}}
$$

where the computation of $n^{*}$, the mean motion, is given by

$$
n^{*}=\left|\frac{\mathrm{G}^{*}}{\mathrm{a}^{*}}\right|^{-1 / 2}
$$

and

$$
\bar{M}^{*}=M_{e}\left[1+\frac{M^{*}}{M_{e}}\right]
$$

Details of the contribution of each tidal constituent and its associated uncertainty to the secular change of the moon's mean motion is given in Table 11. The total acceleration from the GEM-T1 ocean tidal model is the sum of each of these contributions and, as shown in Table 12 , 
produces a value of $-25.27 \pm 0.6 \mathrm{arcsec}_{\mathrm{cy}}{ }^{2}$ for the secular change in the moon's mean motion. This value is in excellent agreement with latest value obtained observationally by Lunar Laser Ranging: (LLR) (Newhall et al., 1986 in press) of $-24.9 \pm 1$ arcsec/cy ${ }^{2}$. The $\dot{n}_{m}$ observed from LLR is in harmony with the energy dissipation calculated from the earth's ocean tidal model for the earth/moon system. This confirms that energy dissipation due to the ocean tides is the dominant source of the lunar orbit's secular acceleration and no other physical mechanisms are required at the current .6 arcsec $c y^{-2}$ level of precision.

Table 11 also reveals that the long period tides have secular effects on the lunar mean motion. Our formulation allows us to evaluate these effects: the coefficients describing the tides are equatorially referenced whereas the satellite orbital frequencles of the tidal disturbing potential are best described and implemented herein as 1 inear combinations of the ecliptic rates. Previous derivations have missed this phenomenon because of their expansion within an equatorial reference system. In this equatorial system, the correspondence between the tidal perturbation rates and the orbit element rates do not exactly cancel, whereas the more correct derivation (given in Appendix 2) find them doing so, giving rise to secular effects.

In our calculations we have 1 gnored any contribution that arises from the dissipation of energy due to tides raised on the moon by the earth. While $M_{2}$ is not of consequence due to the permanent bulge it causes in the moon's shape, contributions from $\mathrm{O}_{1}$ and $\mathrm{N}_{2}$ may need to be considered. Lambeck (1975) estimates that these terms have small effects, being no more than $.16 \mathrm{~m} / \mathrm{cy}$ in $\frac{\mathrm{da}}{\mathrm{dt}}$ for the moon. This effect is of the order of the accuracy we are now achieving and requires further investigation. 
5.0 TIDAL BRAKING OF THE EARTH'S ROTATION

The ocean tides retard the axial rotation of the earth. Conservation of angular momentum in the earth/moon/sun system requires that the secular change in the rotational velocity of the earth due to the lunisolar tidal torques is (Bursa, 1986):

$$
\begin{aligned}
\dot{\Omega}= & A_{1} \frac{d n_{m}}{d t}+A_{2} \frac{d e_{m}}{d t}+A_{3} \frac{d i_{m}}{d t} \\
& +B_{1} \frac{d n_{s}}{d t}+B_{2} \frac{d e_{s}}{d t}+B_{3} \frac{d i}{d t}-\frac{\Omega}{C} \frac{d C}{d t}
\end{aligned}
$$

where $a_{m}, e_{m}$, $i_{m}$ are the ecliptic Keplerian elements of the moon; $a_{s}, e_{s}, i_{s}$ are the equatorial Keplerian elements of the apparent sun; the A terms are for the orbital angular momentum contributions of the Earth/Moon system; the B terms are for the orbital contributions of the Earth/Moon system about the sun; and the $C$ term represents the effects due to changes in the Earth's polar moment of inertia $C$.

The formulae for the A and B terms, which are taken from Lambeck (1980), have been derived from conservation of angular momentum in the direction of the Earth's mean spin axis. In Lambeck's derivation, he uses the inclination of the lunar orbit on the equator a veraged over one period of the lunar ascending node on the ecliptic, $I_{m}$. Thus

$$
\cos I_{m}=\cos i_{s} \cos i_{m}
$$


The requisite formulae are then, where $M$ is the mass of the Earth, $M_{S}$ is the mass of the Sun, and $M_{m}$ is the mass of the Moon:

$$
\begin{aligned}
& F_{m}=\left.\left|\begin{array}{cc}
M & M_{m} \\
M+M_{m}
\end{array}\right|\right|_{-} ^{n_{m} a_{m}^{2} \cos i_{s}} \frac{F_{m}}{C} \cos i_{m} \\
& A_{1}=\left.\frac{m}{3 n_{m}}\right|_{2}=F_{m} e_{m} \cos i_{m} \\
& A_{3}=F_{m} \sin i_{m}
\end{aligned}
$$

for the lunar terms, and

$$
\begin{aligned}
& F_{s}=\left.\left|\frac{M_{s}\left(M+M_{m}\right)}{M_{s}+M+M_{m}}\right|\right|_{-} ^{n_{s}} \frac{a_{s}^{2}}{C} \mid \\
& B_{1}=\frac{F_{s}}{3 n_{s}} \cos i_{s} \\
& B_{2}=F_{s} e_{s} \cos i_{s} \\
& B_{3}=F_{s} \sin i_{s}
\end{aligned}
$$

for the solar terms. 
Bursa (1986) has evaluated the effects due to changes in the Earth's polar moment of inertia due to the secular time variations in the second zonal harmonic:

$$
\frac{\mathrm{dC}}{\mathrm{dt}}=\frac{2}{3} \mathrm{Ma}^{2} \frac{\mathrm{dJ}}{\mathrm{dt}} \quad \mathrm{kg} \mathrm{m} \mathrm{m}^{2} / \mathrm{cy}
$$

where $\mathrm{Ma}^{2}$ is $2.4296 \times 10^{38} \mathrm{~kg} \mathrm{~m}$. The corresponding $\mathrm{C}$ is $8.0378 \times 10^{37} \mathrm{~kg} \mathrm{~m} \mathrm{~m}^{2}$ and $\Omega$ is $7.292115 \times 10^{-5} \mathrm{rad} / \mathrm{sec}$. Yoder et al (1983) have estimated:

$$
\frac{\mathrm{dJ}}{\mathrm{dt}}=(-2.8 \pm 0.6) \times 10^{-9} / \mathrm{cy}
$$

from Lageos tracking data, and have attributed it to the effects of post-glacial rebound. Thus, the non-tidal acceleration of the earth is

$$
\begin{aligned}
\dot{\Omega}_{\mathrm{NT}} & =-\frac{\Omega}{\mathrm{C}} \frac{\mathrm{dC}}{\mathrm{dt}} \\
& =(1.29 \pm .28) \times 10^{-22} \mathrm{rad} / \mathrm{sec}^{2}
\end{aligned}
$$

Each second degree tide in the GEM-T1 expansion, including both adjusted and modeled values, has been used to calculate its contribution to braking the earth's rotation. These rates with their associated uncertainties are given in Table 11. A summary of the long period, diurnal and semi-diurnal contributions to $\dot{\Omega}$ and that from the non-tidal secular change in $J_{2}$ is presented in Table 12. Our estimate of the secular tidal acceleration of the earth's rotation $\left(\dot{\Omega}_{\mathrm{T}}\right)$ is $-5.98 \pm$ $0.22 \times 10^{-22} \mathrm{rad} \mathrm{sec}{ }^{-2}$. Taking into account the non-tidal effect of the change in the earth's oblateness $\left(j_{2}\right)$, our best estimate of the secular braking of the earth's rotation is $-4.69 \pm 0.36 \times 10^{-22} \mathrm{rad} \mathrm{sec}^{-2}$. In terms of the change in length of day, $\dot{\Omega}_{\mathrm{T}}$ corresponds to $2.25 \pm .08$ msec $\mathrm{cy}^{-1}$ and $\dot{\Omega}$ corresponds to $1.76 \pm .14 \mathrm{msec} \mathrm{cy}^{-1}$.

Lambeck (1980) summarizes the astronomical results which have
been obtained for constraining the values of $\dot{\Omega}$ and $\dot{n}_{m}$ and for the 
determination of $\dot{\Omega}$ itself. Although there appears to be a wide variation of values, Lambeck found $\dot{\Omega}=-5.48 \times 10^{-22} \mathrm{rad} \mathrm{s}^{-2}$ to be his consensus value from astronomical observations. Our estimate of this rate, which was satellite derived using both a new ocean tidal model and the observed non-tidal change in the earth's and zonal harmonic, agrees reasonably well with his interpretation of the astronomical constraints. Clearly, this new satellite determination lacks many of the problems associated with the determination of the time and place of ancient eclipses, and represents a significant advancement in determining the tidal braking of the earth's axial rotation.

The derived value for $\dot{\Omega}$ discussed herein, is solely based on the long term secular effect. While the tidal contribution, $\dot{\Omega}_{\mathrm{T}}$, is complete and not expected to vary over the three thousand years of recent history (Lambeck, 1980), the non-tidal contributions may not be complete. In our derivation of $\dot{\Omega}_{\mathrm{NT}}$ we have based the estimate on the effects which arise from $j_{2}$. Yoder et al (1983) suggest that other smaller contributions may come, for example, from $j_{3}$, although present orbital histories cannot accurately resolve them.

Recently, a re-analysis of the eclipse records by Stephenson and Morrison (1984) suggests that there is evidence of a non-uniform decrease in the earth's rotation rate over the last three thousand years. However, the physical basis for any such change remains unexplained. In the case of Stephenson and Morrison, the evidence for a changing non-tidal acceleration is based upon an apparent discrepancy between the historical eclipse results prior to 1620 and the analysis of the later telescopic data subsequent to 1620. From their telescopic data, they derive changes in the length of day of $1.4 \mathrm{msec} \mathrm{cy}^{-1}$ versus $2.4 \mathrm{msec} \mathrm{cy}^{-1}$ for the earlier eclipse data. The artificial satellite results for $\dot{\Omega}_{\mathrm{T}}$, the $t i d a l$ effect alone, yleld $2.25 \pm .1 \mathrm{msec} \mathrm{cy}^{-1}$, which is a value quite consistent with that Stephenson and Morrison found from their early eclipse records. Our value of $1.76 \pm .14$ for $\dot{\Omega}$ is not really 
too different from their 1.4 given the accuracies of the astronomic analyses. Their telescopic results depart from those found herein using modern satellite laser ranging techniques by about -.4 , and the early eclipse results depart by about .6. A simple explanation for these disagreements may be that the astronomic data is of insufficient accuracy; also error sources such as inconsistent reference frames may be present. Given the lack of supporting error analyses, their conclusion of non-uniformity in the decrease of the earth's rotation rate is questionable.

From another perspective, Lambeck (1980, eqn. 10.5.2) estimated that the tidal deceleration of the earth's rotation and the secular change in the lunar mean motion are related by

$$
\dot{\Omega}_{\mathrm{T}}=(51 \pm 4) \dot{\mathrm{n}}_{\mathrm{m}}
$$

This is simply derived from the separate estimates of $\dot{n}_{\mathrm{m}}$ and $\dot{\Omega}_{\mathrm{T}}$. If we use this equation with our estimate of $\dot{n}_{m}$, we find $\dot{\Omega}_{T}=-6.27 \times 10^{-22}$ $\mathrm{rad} / \mathrm{sec}^{2}$, which is in good agreement (one sigma) with our solution. Our estimates of $\dot{n}_{\mathrm{m}}$ and $\dot{\mathrm{n}}_{\mathrm{T}}$ provide the corresponding relationship

$$
\dot{\Omega}_{\mathrm{T}}=(49 \pm 3) \dot{n}_{\mathrm{m}}
$$

indicating that we find the Lambeck formula slightly overestimates the effect of $\dot{n}_{\mathrm{m}}$ on $\dot{\Omega}_{\mathrm{T}}$.

These results from the present artificial satellite analyses represent the $\dot{\Omega}$ and $\dot{n}_{m}$ of an epoch around 1980 . These results can be directly compared to the excellent contemporary observation history of the earth's rotational behaviour available from the space technologies of Satellite and Lunar Laser Ranging and Very Long Baseline Interfercmetry to assess the contemporary role tides play in this complex retardation of the earth's rotation rate. 



\subsection{SUMMARY}

Modern laser tracking technologies allow us to monitor the orbital evolution of near-earth satellites to unprecedented levels of accuracy. Recently acquired observation data sets from a total of seventeen mostly laser satellites have been used to develop detailed models of the forces which explain these observed orbital effects. Both the time invariant and the temporally varying tidal gravitiational potential fields of the earth have been accurately resolved from our analysis of direct satellite tracking observations. This model, Goddard Earth Model (GEM)$T 1$, is the most complete gravitational and dynamic tidal solution ever produced exclusively from direct satellite tracking data.

The GEM-T1 solution simultaneously recovered a complete gravitational field to degree and order 36 and 66 tidal coefficients representing twelve major tides. The solution made use of modern geodetic constants, such as the J2000 Reference System complete with the wahr nutation and solid earth tidal models. A more complete model of the background ocean tides was used, which had been developed to reduce aliasing in the recovery. These unadjusted tidal terms included over 550 coefficents representing the longest wavelength constituents of 32 major and minor tides. The minor ocean tidal models were estimated using a predictive technique based on the observed major tides and a postulated linearity of the admittances. The tidal perturbations which result from the separate solid earth, atmosphere and oceans give rise to identical orbital frequencies which cannot be separately resolved from an analysis of orbital motion. However, the total tidal effect is accurately sensed in these well tracked orbits and the tidal perturbations are known to be strong.

The complete GEM-T1 tidal model containing both the adjusted and unadjusted terms has been used to estimate the effects of the earth's tides on the earth/moon/sun system. The secular change in the lunar mean 
motion estimated from this dynamic tidal model, $-25.27 \pm .61 \operatorname{arcsec} \mathrm{cy}^{-2}$, agrees very well with the value of $-24.9 \pm 1$. arcsec $c y^{-2}$ recovered from a decade of Lunar Laser Ranging by Newhall et al, 1986. The tidal braking of the rate of the earth's rotation due to conservation of angular momentum in the earth/moon/sun system yields a value of $-5.98 \pm .22 \times 10^{-22} \mathrm{rad} \mathrm{sec}^{-2}$, corresonding to $2.25 \pm 0.08 \mathrm{msec} / \mathrm{cy}$ in length of day. Thus we have derived a set of tides from tracking of artificial satellites which seems to consistantly predict the observed evolution of the lunar orbit.

Taking into account the non-tidal braking due to the Lageos derived secular rate of change in the earth's second zonal harmonic (Yoder et al, 1983), artificial satellite techniques provide a total value for the secular change in the earth's rotation rate of $-4.69 \pm .36 \times 10^{-22} \mathrm{rad} \mathrm{sec}^{-2}$, which corresponds to $1.76 \pm .14 \mathrm{msec} / \mathrm{cy}$ in length of day. This value is consistent with recent astronomic studies of eclipse records such as that of Stephenson and Morrison (1984), who obtained $2.4 \mathrm{msec} / \mathrm{cy}$ for the data prior to 1620 and 1.4 msec/cy for the telescopic data since 1620. Because there may exist other secular effects which we have not yet accounted for, and because of the significantly poorer accuracies acheivable from the astromical data, this agreement must be regarded as quite good. Also, the $1.76 \mathrm{msec} / \mathrm{cy}$ does not confirm any inference of a non-uniform change in the earth's rotation rate; rather it tends to indicate that the difference of $1 \mathrm{msec} / \mathrm{cy}$ in the separate estimates of Stephenson and Morrison may reflect the separate errors of their determinations. 
REFERENCES

Balmino, G., "Analytical Expressions for Earth Tides Perturbations on Close Earth Satellites," The Use of Artificial Satellites for Geodesy and Geodynamics, pp. 313-322, edited by G. Veis, National Technical University, Athens, Greece, 1974.

Born, G.H., Stewart, R.H, and Yamarone, C.A., "TOPEX - A Spaceborne ocean Observing System," Monitoring Earth's Ocean, Land, and Atmosphere from Space--Sensors, Systems, and Applications, Vol. 97 of Progress in Astronautics and Aeronautics series, pp. 464-479. Published by the American Institute of Aeronautics and Astronautics, Inc., New York, 1985.

Bursa, M., "Geodynamics Due to the Varying Second Zonal Geopotential Harmonic", paper presented at the XXVI COSPAR Conference, Toul ouse, France, June, 1986.

Cazenave, A., Daillet, S., "Lunar Tidal Acceleration from Earth Satellite Orbit Analyses", J. Geophys. Res., 86(B3), 1659-1663, 1981.

Christodoulidis, D.C., Smith, D.E., Klosko, S.M., Torrence, M.H., Dunn, P.J., "A GSFC Alternative to the SLR MERIT Constants", Proceedings of the International Conference on Earth Rotation and the Terrestrial Reference Frame, July 31-August 2, 1985.

Christodoulidis, D.C., Williamson, R., Chinn, D., and Estes, R., "On the Prediction of Ocean Tides for Minor Constituents", Proceedings of the Tenth International Symposium on Earth Tides, R. Vieira editor, Consejo Superior de Investigaciones Cientificas, Madrid, 1986.

Christodoulidis, D.C., Smith, D.E., Klosko, S.M., and Dunn, P.J., "Solid Earth and Ocean Tide Parameters From LAGEOS", Proceedings of the Tenth International Symposium on Earth Tides, R. Vieira editor, Consejo Superior de Investigaciones Cientificas, Madrid, $1986 \mathrm{~b}$. 
Coates, R.J., Frey, H., Mead, G.D., Bosworth, J.M., "Space-Age Geodesy: The NASA Crustal Dynamics Project", IEEE Transactions on Geoscience and Remote Sensing, Vol GE-23, No. 4, July 1985.

Dehant, V., "Tidal Parameters for an Inelastic Earth", paper presented at the European Geophysical Society Joint Meeting, Kiel, West Germany, August, 1986a.

Dehant, V., "Comparison Between the Theoretical and Observed Tidal Gravimetric Factors", paper presented at the European Geophysical Society Joint Meeting, Kiel, West Germany, August, $1986 \mathrm{~b}$.

Dehant, V. "Integration of the Gravitational Motion Equations for an Elliptical, Uniformly Rotating Earth with an Inelastic Mantle", paper presented at the European Geophysical Society Joint Meeting, Kiel, West Germany, August, $1986 \mathrm{c}$.

Doodson, A.T., "The Harmonic Development of the Tide Generating Potential," Proc. Roy. Soc., London, 100, 305, 1921.

Douglas, B.C., Klosko, S.M., Marsh, J.G., and Williamson, R.G., "Tidal Parameters from the Variation of Inclination of GEOS-1 and GEOS2", Celestial Mechanics, (10), 165-178, 1974.

Felsentreger, T.L., Marsh, J.G., Williamson, R.G., "M2 Ocean Tide Parameters and the Deceleration of the Moon's Mean Longitude from Satellite Orbit Data", J. Geophys.Res., 84(B9), 4675-4679, 1979.

Felsentreger, T.L., Marsh, J.G., Agreen, R.W., "Analyses of the Solid Earth and Ocean Tidal Perturbations on the Orbits of the GEOS-1 and GEOS-2 Satellites", J. Geophys.Res., 81(14), 2557- 2563, 1976. 
Goad, C.C., Douglas, B.C., "Lunar Tidal Acceleration Obtained from Satellite-derived Ocean Tide Parameters", J. Geophys. Res., $83(85), 2306-2310,1978$.

Goad, C.C., Douglas, B.C., "Determination of M2 Ocean Tide Parameters from Satellite Orbit Perturbations", J. Geophys. Res., 12(5), 1977.

Goad, C.C., "Application of Digital Filtering to Satellite Geodesy", NOAA Technical Report NOS 71, NGS 6 Rockville, MD., May 1977. (Doctoral dissertation, School of Engineering and Architecture, Catholic University of America, Washington, D.C.)

Kaula, W.M., "Tidal Dissipation by Solid Friction and the Resulting Orbital Evolution", Reviews of Geophysics, Vol. 2, No.4, November 1964.

Kaula, W.M., Theory of Satellite Geodesy, Blaisdell Publishing Company, Waltham, Mass., 1966.

Kaula, W.M., "Tidal Friction with Latitude-Dependent Amplitude and Phase Angle", Astronomical Journal, 74, No. 9, November 1969.

Lambeck, K., Cazenave, A., Balmino, G., "Solid Earth and Ocean Tides Estimated from Satellite orbit Analyses", Rev. Geophys., 12, 421434, 1974.

Lambeck, K., "Effects of Tidal Dissipation in the Oceans on the Moon's Orbit and the Earth's Rotation", J. Geophys. R., $80(20), 1975$.

Lambeck, K., "Tidal Dissipation in the Oceans: Astronomical, Geophysical and Oceanographic Consequences", Philosophical Transactions of the Royal Society of London, Vol. 287, No. 1347, 545-594, December $19,1977$. 
Lambeck, K., The Earth's Variable Rotation: Geophysical Causes and Consequences, Cambridge University Press, New York, 1980.

Marsh, J.M., "An Improved Model of the Earth's Gravitaitonal Field: GEMT1", NASA Technical Report, in press, 1987.

Melbourbe, W., Anderle, R., Feissel, M., King, R., MCCarthy, D., Smith, D., Tapley, B., Vincente, R., "Project MERIT Standards", Circ. 167, U.S. Nav. Observ., Washington, D.C., 1983.

Newhall, X.X., Williams, J.G., Dickey, J.0., "Earth Rotation from Lunar Laser Ranging", JPL Geodesy and Geophysics Preprint, No. 153, December 1986.

Parke, M.E., "01, P1, N2 Models of the Global Ocean Tide on an Elastic Earth Plus Surface Potential and Spherical Harmonic Decompositions for M2, S2, and K1", Marine Geodesy, Vol. 6, No. 1, 1982.

Schwiderski, E.W., "Atlas of Ocean Tidal Charts and Maps, Part I: The Semidiurnal Principal Lunar Tide M2", Marine Geodesy, Vol. 6, No. $3-4,1983$.

Schwiderski, E.W., Global Ocean Tides, PartV: The Diurnal Principal Lunar Tide (01), Atlas of Tidal Charts and Maps, May 1981.

Schwiderski, E.W., "On Charting Global Ocean Tides", Reviews of Geophysics and Space Physics, Vol. 18, No. 1, 243-268, February 1980. 
Stephenson, F.R., Morrison, L.V., "Long-term Changes in the Rotation of The Earth: 700 B.C. to A.D. 1980", Phil.Trans. R. Soc. Lond, A $313,47-70,1984$.

Van Flandern, T.C., "A Determination of the Rate of Change of G", Mon. Not. R. Astron. Soc., 170, 333-342, 1975.

Wahr, J., Bergen, Z., "The Effects of Mantle Anelasticity on Nutations, Earth Tides, and Tidal Variations in Rotation Rate" in press.

Wahr, J.M., "Body Tides on an Elliptical Rotating, Elastic, and Oceanless Earth", Geophys. J.R. Astron. Soc., 64, 677-703, 1981.

Wahr, J.M., "The Tidal Motions of a Rotating, Elliptical, Elastic and oceanless Earth", PhD. Thesis, University of Colorado, 1979.

Williamson, R.G., Marsh, J.G., "Starlette Geodynamics: The Earth's Tidal Response", J. Geophys. R., 90(B11), 1985.

Williamson, R.G. and B.C. Douglas, HAP User;s Guide, Wolf Research and Development Corporation, Report No. NAS-11726-163, 1972.

Yoder, C.F., et al, "Secular Variation of Earth's Gravitational Harmonic J2 Coefficient from LAGEOS and Nontidal Acceleration of Earth Rotation", Nature, 303, 757-762, 1983.

Zschau, J., "Tidal Friction in the Solid Earth: Constraints from the Chandler Wobble Period", Space Geodesy and Geodynamics, Anderson, A. and Cazenave, A. Editors, Academic Press, 1986.

Zschau, J., "Anelasticity in the Earth's Mantle: Implications for the Frequency Dependence of Love Numbers", in press, 1985. 


\section{TABLE 1.}

\begin{tabular}{|c|c|c|c|}
\hline $\begin{array}{l}\text { Darwinian } \\
\text { Symbol }\end{array}$ & $\begin{array}{l}\text { Doodson's } \\
\text { Argument } \\
\text { Number } \\
\end{array}$ & $\begin{array}{l}\text { Period } \\
(\mathrm{hr})\end{array}$ & Description \\
\hline$M_{2}$ & 255.555 & 12.42 & Principal lunar semidiurnal \\
\hline$S_{2}$ & 273.555 & 12.00 & Principal solar semidiurnal \\
\hline $\mathrm{N}_{2}$ & 245.655 & 12.66 & $\begin{array}{l}\text { Larger lunar elliptic } \\
\quad \text { semidi urnal }\end{array}$ \\
\hline $\mathrm{K}_{2}$ & 275.555 & 11.97 & Lunar/Solar semidiurnal \\
\hline $\mathrm{L}_{2}$ & 265.455 & 12.19 & Smaller lunar elliptic \\
\hline $\mathrm{K}_{1}$ & 165.555 & 23.93 & Lunar/Solar diurnal \\
\hline$o_{1}$ & 145.555 & 25.82 & Principal lunar diurnal \\
\hline$P_{1}$ & 163.555 & 24.07 & Principal solar diurnal \\
\hline$M_{f}$ & 075.555 & $13.66 d$ & Lunar fortnightly \\
\hline$M_{m}$ & 065.455 & $27.55 d$ & Lunar monthly \\
\hline$s_{\text {sa }}$ & 057.555 & $188.62 d$ & Solar semi-annual \\
\hline
\end{tabular}


TABLE 2. WAHR LOVE NUMBERS MODELED IN GEM-T1

\begin{tabular}{|c|c|c|}
\hline Band & Iidal Line (f) & $k_{2, f}$ \\
\hline Long Period & All & .299 \\
\hline \multirow[t]{6}{*}{ Di urnal } & $145555(01)$ & .298 \\
\hline & $163555(P 1)$ & .287 \\
\hline & 165545 & .259 \\
\hline & 165555 (K1) & .256 \\
\hline & 165565 & .253 \\
\hline & 166554 (PSI) & .466 \\
\hline Semi-Diurnal & All & .302 \\
\hline
\end{tabular}

*A1l phases are equal to zero. 


\section{OCEAN TIDE MODELING \\ FOR \\ GRAVITY RECOVERY}

- LONG PERIOD TIDES •

\begin{tabular}{c}
$\begin{array}{c}\text { Doodson } \\
\text { No. }\end{array}$ \\
\hline 056.554 \\
057.555 \\
058.554 \\
065.455 \\
075.555 \\
075.565
\end{tabular}

I 35.655

145.545

145.555

155.455

155.655

162.556

163.555

164.556

165.545

165.555

165.565

166.554

167.555

175.455

185.555
Dorwin

Nome

S.

S 80

$M_{\text {f }}$
Modeled

deg. $2 \rightarrow 6$

prograde

only

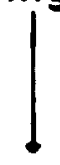

Adjusted

deg. 2

deg. 2

none

deg. 2

deg. 2

none

- DIURNal •

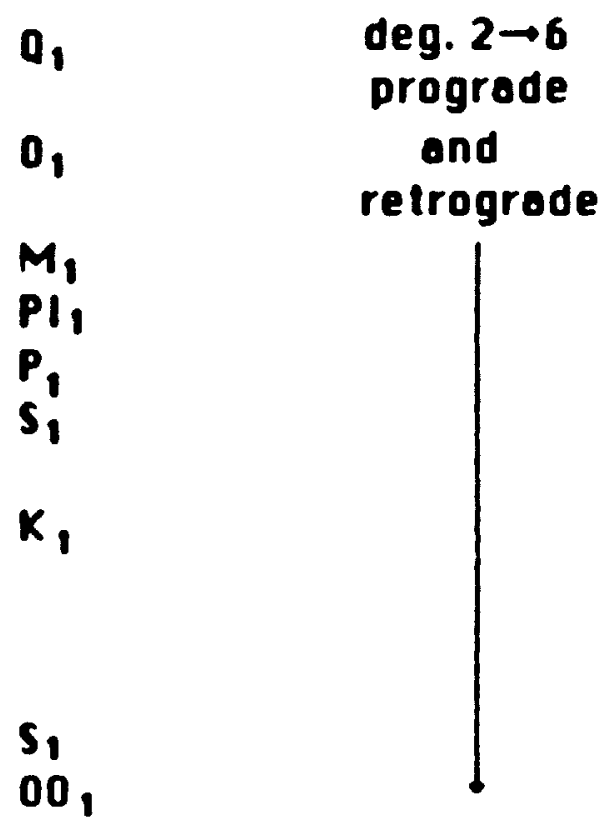

none
none
deg. $2,3,4$
none
none
none
deg. $2,3,4$
none
none
deg. $2,3,4$
none
none
none
none
none




\section{- SEMI-DIURNAL •}

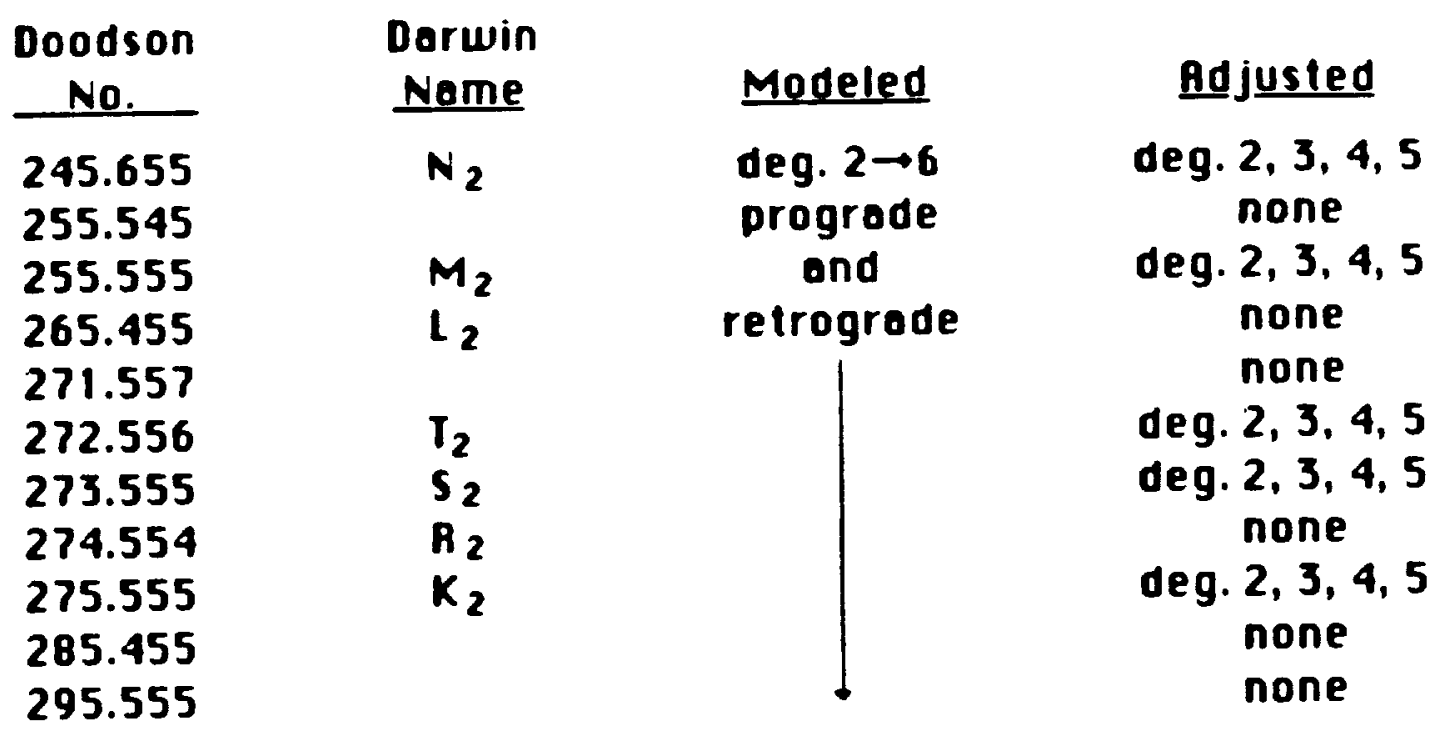


$\triangle P R I O R \perp G R A Y I Y Y M O D E L$

$\begin{array}{lll}\text { GEM-L2 } & \text { FOR } & \text { LAGEOS } \\ \text { PGS-1331. } & \text { FOR } & \text { STARLETIE } \\ \text { PGS-S4' } & \text { FOR } & \text { SEASAT } \\ \text { GEM-10B' } & \text { FOR } & \text { ALL OTHER SATELLITES }\end{array}$

PRIME ( ) INOICATES FIELDS RESOL VED HOLOING C, $S_{2,1} \equiv 0$

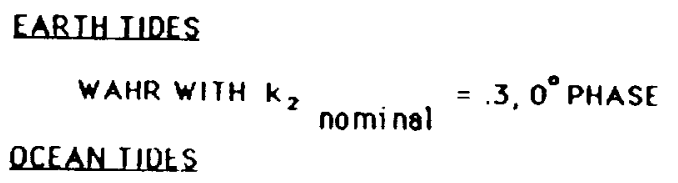

CHRISTODOULIDISET AL MODEL WITH 600 INDIVIDUAL IERMS, PREDICTED FROM OCEANOGRAPHIC MODELS USING ADMITTANCES

IIDAL DEFORMATIONS

$h_{2}$ AND $h_{2}$ AT MERIT VALUES

GM. o. .111

$$
\begin{aligned}
G M & =398600.436 \mathrm{~km}^{3} / \mathrm{sec}^{2} \\
\theta_{f} & =6378.137 \mathrm{~km} \\
I_{f} & =298.257
\end{aligned}
$$

\section{POLAR MOTION AND AI-UTI}

ZERO MEAN SET OF POLAR MOTIONS BASED ON 6 YEARS OF SLR DETERMINATIONS

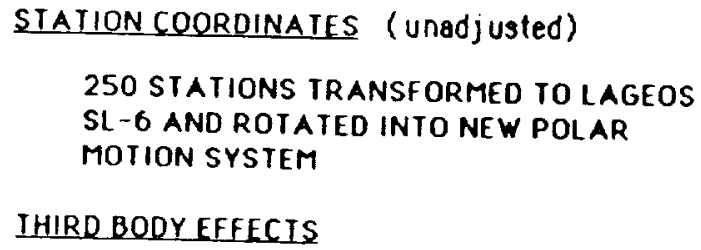




\section{DATA OTILIZED II PRELIYIMART} TOPEI GRAPITT ODEL: 1986

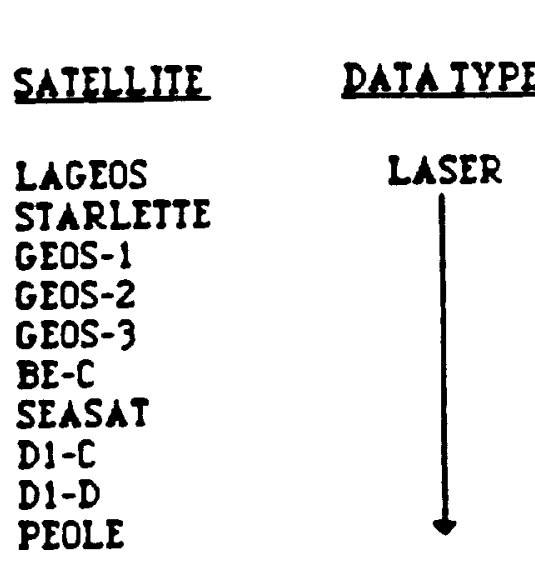

NUMBER OF
NORMAL MATRICES
S6
46
48
28
36
39
14
4
6
6

NUMBER OF

SUB-TOTAL - LASER

285

OBSERVATIONS

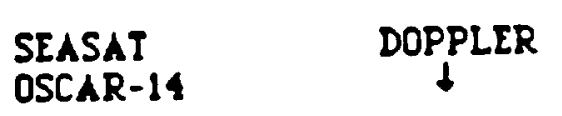

15

13

144527

57356

71287

26613

42407

64240

14923

7455

11487

1113

SUB-TOTAL - DOPPLER

28

$\$ 44.408$

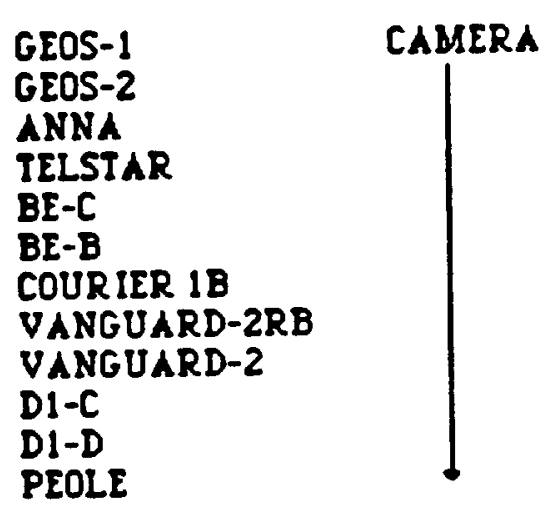

43
46

60750

61403

4463

30

30

50

3962

7501

1739

2476

686

1299

2712

6111

38

SUB-TOTAL - CAMERA

273

153.140

TOTAL

$580^{*}$

798,688

* PEOLE arcs contained both optical and laser data. 
Table 6

SATELLITE ORBITAL CBARACTERISTICS

\begin{tabular}{|c|c|c|c|c|c|}
\hline $\begin{array}{l}\text { SATELLITE } \\
\text { NAME }\end{array}$ & $\begin{array}{l}\text { SATELLITE } \\
\text { ID NO } \\
\end{array}$ & $\begin{array}{l}\text { SEMI-MA JOR } \\
\quad \text { AXIS } \\
\end{array}$ & $E C C$ & $\begin{array}{l}\text { INCL } \\
\text { (DEG) }\end{array}$ & $\begin{array}{l}\text { DATA* } \\
\text { IYPE }\end{array}$ \\
\hline $\begin{array}{l}\text { ANNA-1B } \\
\text { BE-B } \\
\text { BE-C } \\
\text { COURIER-1B } \\
\text { DI-C } \\
\text { DI-D } \\
\text { GEOS-1 } \\
\text { GEOS-2 } \\
\text { GEOS-3 } \\
\text { LAGEOS } \\
\text { OSCAR } \\
\text { PEOLE } \\
\text { SEASAT } \\
\text { STARLETTE } \\
\text { IELESTAR-1 } \\
\text { VANGUARD-2RB } \\
\text { VANGUARD-2 }\end{array}$ & $\begin{array}{l}620601 \\
640841 \\
650321 \\
600131 \\
670111 \\
670141 \\
650891 \\
680021 \\
750271 \\
760391 \\
670921 \\
701091 \\
780641 \\
750101 \\
620291 \\
590012 \\
590011\end{array}$ & $\begin{array}{l}7501 \\
7354 \\
7507 \\
7469 \\
7341 \\
7622 \\
8075 \\
7711 \\
7226 \\
12273 . \\
7440 \\
7006 \\
7170 \\
7331 \\
9669 \\
8496 \\
8298\end{array}$ & $\begin{array}{l}.0082 \\
.0135 \\
.0257 \\
.0161 \\
.0532 \\
.0848 \\
.0719 \\
.0330 \\
.0008 \\
.0038 \\
.0029 \\
.0164 \\
.0021 \\
.0204 \\
.2429 \\
.1832 \\
.1641\end{array}$ & $\begin{array}{l}50.12 \\
79.69 \\
41.19 \\
28.31 \\
39.97 \\
39.46 \\
59.39 \\
105.79 \\
114.98 \\
109.85 \\
89.27 \\
15.01 \\
108.02 \\
49.80 \\
44.79 \\
32.92 \\
32.89\end{array}$ & $\begin{array}{r}0 \\
0 \\
L .0 \\
0 \\
L .0 \\
L .0 \\
L .0 \\
L .0 \\
L \\
L \\
D \\
L .0 \\
D . L \\
L \\
0 \\
0 \\
0\end{array}$ \\
\hline
\end{tabular}

\footnotetext{
- De Doppler

$L$ L Laser

$0:$ Optical
} 


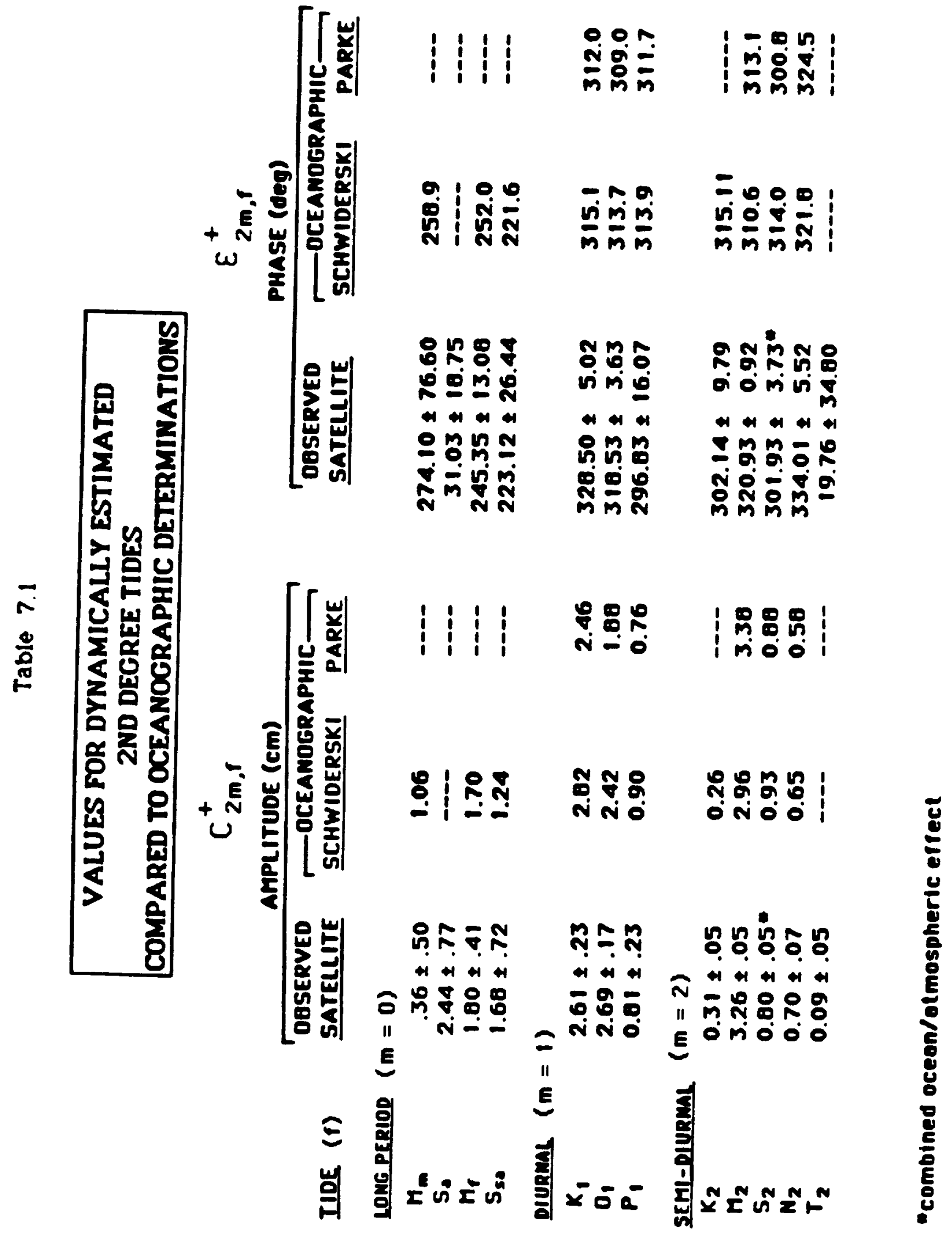




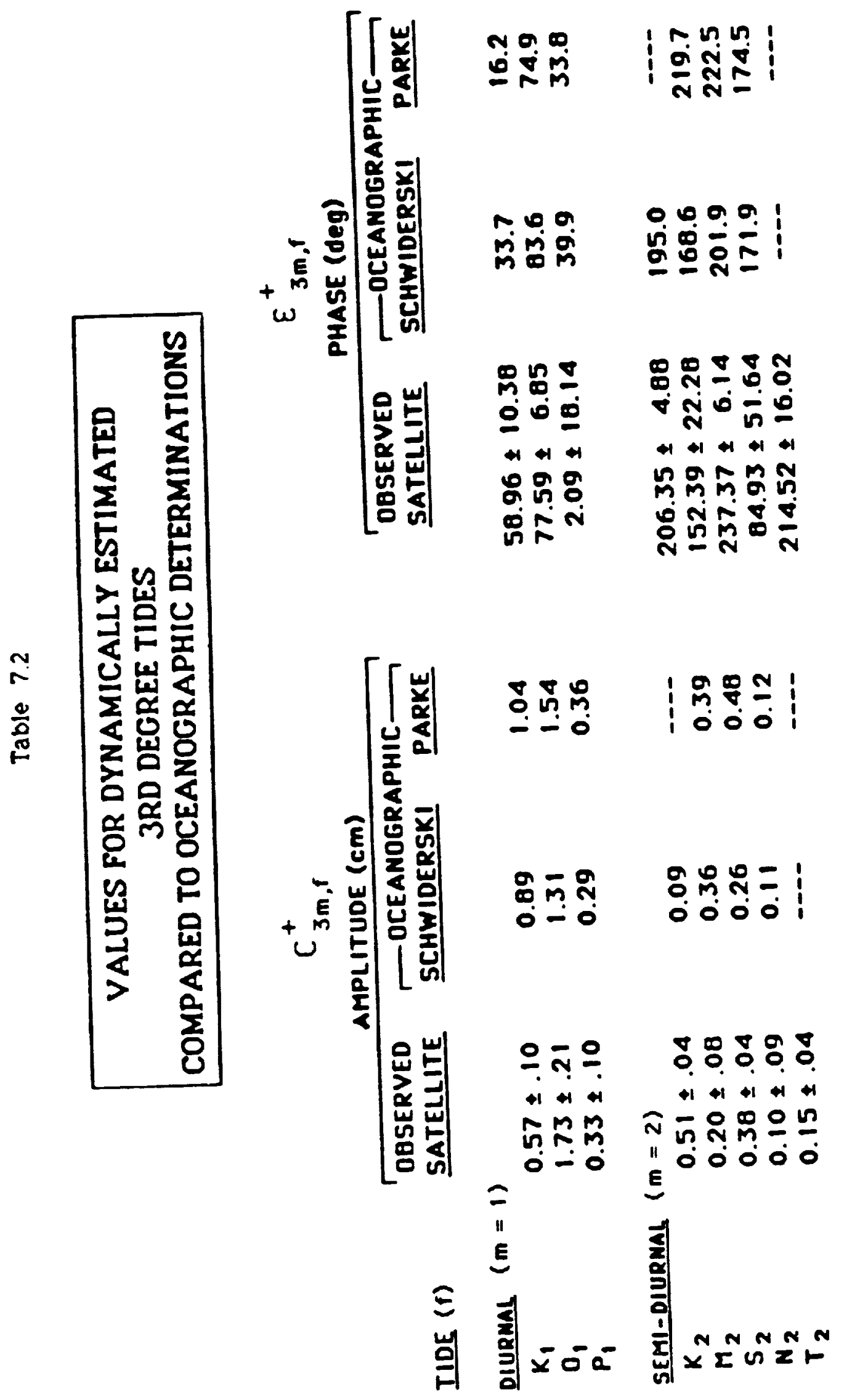




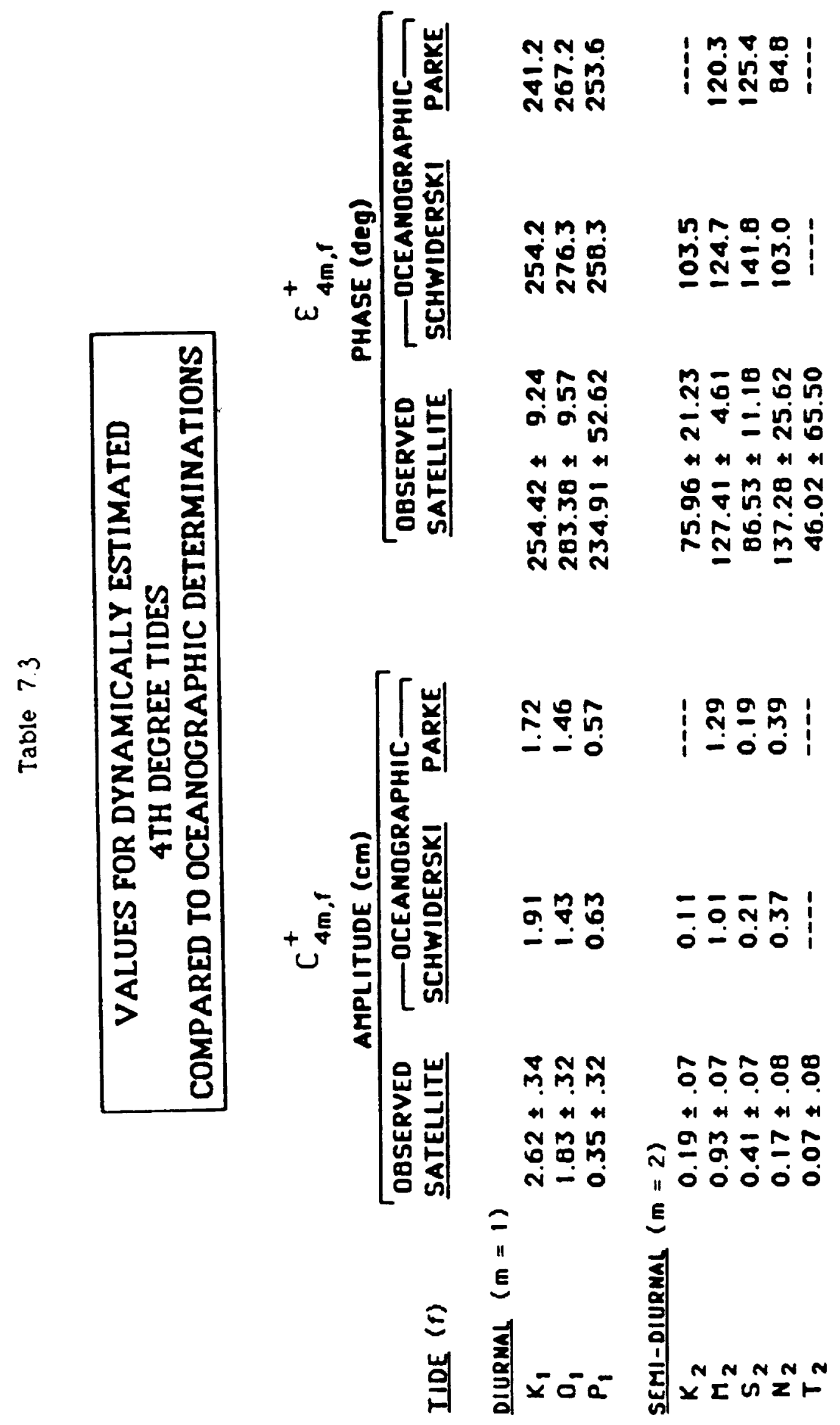




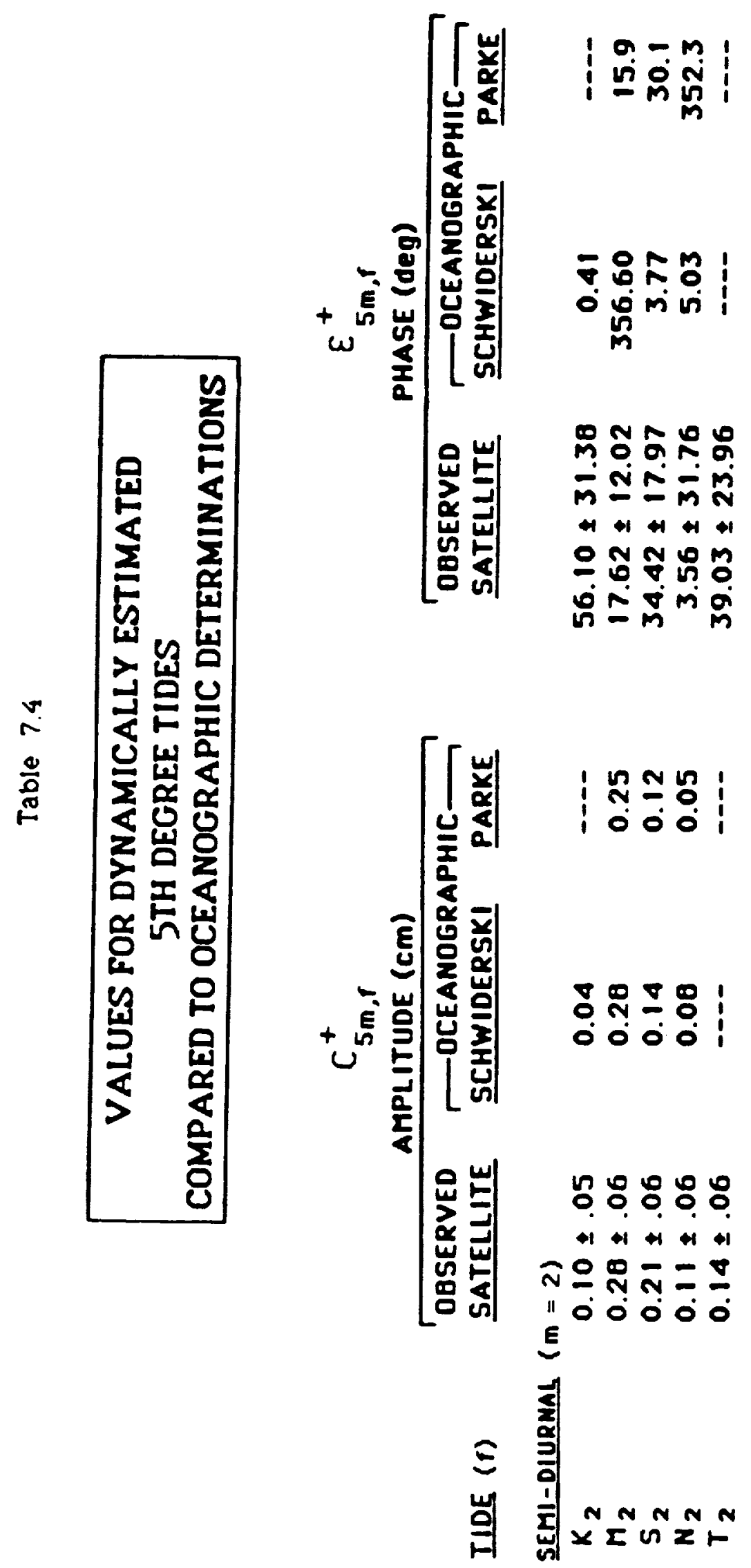


Table 8

COMPARISON OF OCEAN TIDAL COEFFICIENTS

FROM

SATELLITE SOLUTIONS

\begin{tabular}{|c|c|c|c|c|c|}
\hline \multicolumn{2}{|c|}{$\begin{array}{l}\text { Amp } \\
C_{2 m}^{+} \\
\end{array}$} & $\begin{array}{c}\text { Phose } \\
E+2 m \\
\end{array}$ & $\begin{array}{l}\text { Amp } \\
C^{+} \\
\end{array}$ & $\begin{array}{l}\text { Phase } \\
E^{+}{ }_{4 m} \\
\end{array}$ & $\begin{array}{l}\text { Source } \\
\text { (ref) } \\
\end{array}$ \\
\hline$M_{m}$ & $\begin{array}{l}0.36 \\
1.99\end{array}$ & $\begin{array}{l}274.10 \\
132.5\end{array}$ & & & $\begin{array}{l}\text { GEM-I I (lhis } \\
\text { paper) } \\
\text { STARLETTE (B) }\end{array}$ \\
\hline$M_{r}$ & $\begin{array}{l}1.80 \\
1.70\end{array}$ & $\begin{array}{l}245.35 \\
265.4\end{array}$ & & & $\begin{array}{l}\text { GEM-TI } \\
\text { STARLETTE (B) }\end{array}$ \\
\hline $0_{1}$ & $\begin{array}{l}2.69 \\
2.83 \\
2.85 \\
2.96\end{array}$ & $\begin{array}{l}318.53 \\
327 \\
344.3 \\
354 .\end{array}$ & $\begin{array}{l}1.83 \\
(1.43) * \\
1.44 \\
1.7\end{array}$ & $\begin{array}{l}283.38 \\
(276.3)^{*} \\
304.5 \\
350.0\end{array}$ & $\begin{array}{l}\text { GEM-TI } \\
\text { LAGEOS (C) } \\
\text { STARLETTE (B) } \\
\text { STARLETTE+GEOS } 3 \text { (D) }\end{array}$ \\
\hline$P_{1}$ & $\begin{array}{l}0.81 \\
0.73 \\
1.77\end{array}$ & $\begin{array}{l}296.83 \\
253 . \\
188.3\end{array}$ & $\begin{array}{l}0.35 \\
(0.63) * \\
0.74\end{array}$ & $\begin{array}{l}234.91 \\
(258.3) \\
264.9\end{array}$ & $\begin{array}{l}\text { GEM-T } 1 \\
\text { LAGEOS (C) } \\
\text { STARLETTE (B) }\end{array}$ \\
\hline$k_{1}$ & $\begin{array}{l}2.61 \\
2.63\end{array}$ & $\begin{array}{l}328.50 \\
311.7\end{array}$ & $\begin{array}{l}2.62 \\
3.40\end{array}$ & $\begin{array}{l}254.42 \\
244.4\end{array}$ & $\begin{array}{l}\text { GEM-TI } \\
\text { STARLETTE (B) }\end{array}$ \\
\hline$N_{2}$ & $\begin{array}{l}0.70 \\
0.59 \\
0.76\end{array}$ & $\begin{array}{l}334.0 \\
346 . \\
322.9\end{array}$ & $\begin{array}{l}0.17 \\
(0.37) \\
0.24\end{array}$ & $\begin{array}{l}137.29 \\
(103.0) \\
192.7\end{array}$ & $\begin{array}{l}\text { GEM-I I } \\
\text { LAGEOS (C) } \\
\text { STARLETTE (B) }\end{array}$ \\
\hline \multirow[t]{3}{*}{$M_{2}$} & $\begin{array}{l}3.26 \\
3.45 \\
2.57 \\
3.5 \\
3.1\end{array}$ & $\begin{array}{l}320.93 \\
319 . \\
319.2 \\
338 . \\
333 .\end{array}$ & $\begin{array}{l}0.93 \\
(1.01)= \\
1.70 \\
1.03 \\
1.03\end{array}$ & $\begin{array}{l}127.41 \\
(124.7)= \\
118.5 \\
79 . \\
78 .\end{array}$ & \multirow{3}{*}{$\begin{array}{l}\text { GEM-TI } \\
\text { LAGEOS (C) } \\
\text { STARLETTE (B) } \\
\text { STARLETTE (E) } \\
\text { STARLETTE + GEOS } 3 \text { (D) } \\
\text { OSCAR, STARLETTE, } \\
\text { GEOS } 3 \text { (F) } \\
\text { GEOS } 3+\text { OSCAR (G) } \\
\text { GEOS } 3 \text { (H) }\end{array}$} \\
\hline & 3.4 & 325 & 1.0 & 124 & \\
\hline & $\begin{array}{l}3.2 \\
2.9\end{array}$ & $\begin{array}{l}331 . \\
332 .\end{array}$ & $\begin{array}{l}0.9 \\
1.0\end{array}$ & $\begin{array}{l}113 . \\
115 .\end{array}$ & \\
\hline$S_{2}^{*}$ & $\begin{array}{l}0.80 \\
0.77 \\
0.78\end{array}$ & $\begin{array}{l}301.93 \\
302 . \\
32.4\end{array}$ & $\begin{array}{l}0.41 \\
(0.21) \\
0.42\end{array}$ & $\begin{array}{c}86.53 \\
(141.8)^{*} \\
94.8\end{array}$ & $\begin{array}{l}\text { GEM-T I } \\
\text { LAGEOS (C) } \\
\text { STARLETTE (B) }\end{array}$ \\
\hline$K_{2}$ & $\begin{array}{l}0.31 \\
0.16 \\
0.83\end{array}$ & $\begin{array}{l}302.14 \\
238 \\
23.4\end{array}$ & $\begin{array}{l}0.19 \\
(0.11)= \\
0.53\end{array}$ & $\begin{array}{c}75.96 \\
(103.5)^{*} \\
96.0\end{array}$ & $\begin{array}{l}\text { GEM-TI } \\
\text { LAGEOS (C) } \\
\text { STARLETIE (B) }\end{array}$ \\
\hline
\end{tabular}

* includes atmospheric tide

* held unadiusted at Schwiderski value

(B) Williamson and Marsh, 1985

(f) Felsentreger et al , 1979

(C) Christodoulidis et al., 1985

(G) Goad and Douglas, 1978A

(D) Cazenave and Daillet, 1981

(H) Goad and Dougles, $1978 B$

(E) Cazenave and Daillet, 1981 
Toble 9.

\section{VALUES FOR MODELED 2ND DEGREE TIDES CALCULATED FROM LINEAR ADMITTANCES}

IIDE (f)

LONG PERIOD $(\mathrm{m}=0)$

\subsection{4}

075.565
$C_{2 m, t}^{+}$

AMPLITUDE (cm)
$\mathcal{E}_{2 \mathrm{~m}, \mathrm{f}}^{+}$

PHASE (deg)

\section{DIURNAL $(m=1)$}

$\begin{array}{llll}135.655 & Q_{1} & 0.53 & 313.70 \\ 145.545 & 0_{1 f} & 0.47 & 313.85 \\ 155.455 & M_{1 f} & 0.06 & 314.03 \\ 155.655 & M_{1} & 0.17 & 314.04 \\ 162.556 & q_{1} & 0.05 & 314.22 \\ 164.556 & S_{1} & 0.02 & 314.26 \\ 165.545 & K_{1 f} & 0.05 & 314.28 \\ 165.565 & K_{1} & 0.36 & 314.28 \\ 166.554 & \psi_{1} & 0.02 & 314.30 \\ 167.555 & Q_{1} & 0.04 & 314.32 \\ 175.455 & J_{1} & 0.13 & 314.60 \\ 185.555 & 0_{1} & 0.06 & 315.08\end{array}$

SEMI-DIURNAL ( $m=2)$

$\begin{array}{lll}255.545 & M_{2 S} & 0.11 \\ 265.455 & L_{2} & 0.07 \\ 271.557 & & 0.002 \\ 274.554 & R_{2} & 0.01 \\ 285.455 & & 0.01 \\ 295.555 & & 0.002\end{array}$

313.85

314.03

314.22

314.26

314.28

314.28

314.30

314.32

315.08
316.82

315.86

314.93

314.57

312.22

308.07 


\section{SECULAR CHANGES IN THE ECLIPTIC KEPLER ELEMENTS OF THE MOON AND THE SUN DUE TO TIDES}
$\frac{d \theta_{0}}{d t}$
$3.73 \pm 0.09$
$\mathrm{mcy}^{-1}$
$\frac{d e}{d t}$
$(1.83 \pm 1.10) \times 10^{-11}$
$y r^{-1}$
$\frac{d i}{d t}$
$(-6.65 \pm 0.53) \times 10^{-10}$
deg $\mathrm{yr}^{-1}$
$\frac{d e}{d t} o$
$(1.43 \pm 0.25) \times 10^{-4}$
$m c y^{-1}$
$\frac{d e}{d t} o$
$(1.3 \pm 9.4) \times 10^{-19}$
$y r^{-1}$
$\frac{d i}{d t} o$
$(-5 \pm 51) \times 10^{-17}$
deg $\mathrm{yr}^{-1}$ 
SECULAR CHANGE IN THE MEAN MOTION OF THE

MOON ( $\dot{n}$, in ARCSEC/CY ${ }^{2}$ ) AND IN THE

ROTATIONAL VELOCITY OF THE EARTH

$\left(\dot{\Omega}\right.$, in $\left.10^{-22} \mathrm{RAD} / \mathrm{SEC}^{2}\right)$ DUE TO TIDES

IIDES

1. LONG PERIOD TIDES

$056.554 \mathrm{~S}_{\mathrm{a}}$

$057.555 \mathrm{~S}_{\mathrm{sa}}$

058.554

$065.455 \mathbf{M m}_{\mathbf{m}}$

$075.555 \mathrm{M}_{\mathrm{f}}$

075.565

TOTAL LP-TIDES $\underline{\mathbf{n}}_{\text {? }}$

$\underline{\sigma}_{\dot{\mathbf{n}}_{\mathrm{q}}}$

$\dot{\Omega}$

$\underline{\sigma}_{\dot{\Omega}}$

0.00

0.00

0.00

0.00

$-0.00$

0.00

0.04

0.03

0.00

$-0.00$

0.00

0.31

0.18

$-0.00$

0.12

$-0.12$

0.07

$-0.56$

0.06

$-0.01$

0.02

$\pm 0.36$

$-0.17$

2. DIURNAL TIDES

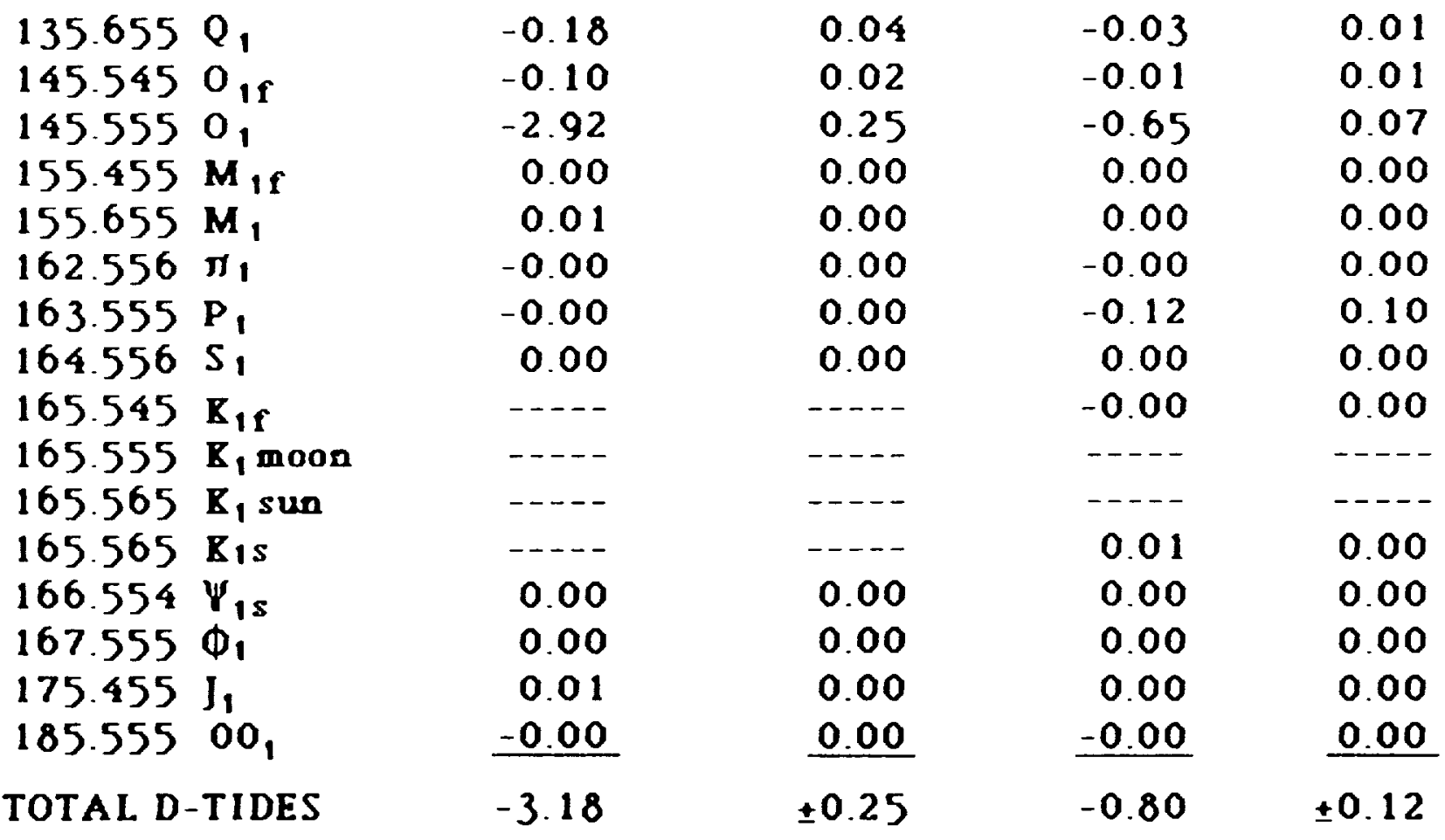




\section{TIDES}

$\underline{\underline{n}}_{a} \quad \underline{\sigma}_{\dot{\mathbf{n}}_{\mathbb{q}}}$

$\dot{\underline{\Omega}}$

$\underline{0}_{\boldsymbol{\Omega}}$

3. SEMI-DIURNAL TIDES

$245.655 \mathbf{N}_{2}$

$255.545 \mathrm{M}_{2 \mathrm{~S}}$

$255.555 \mathrm{M}_{2}$

$265.455 \mathrm{~L}_{2}$

271.557

$272.556 \mathrm{~T}_{2}$

$273.555 \mathrm{~S}_{2}$

$274.554 \mathrm{R}_{2}$

$275.555 \mathrm{~K}_{2} \operatorname{moon}$

$275.555 \mathrm{~K}_{2}$ sun

285.455

295.555

TOTAL SD-TIDES
$-1.43$

0.02

$-20.00$

0.01

$-0.00$

$-0.00$

$-0.00$

0.00

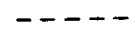

$-0.00$

0.00

$-21.40$
0.16

0.01

0.40

0.00

0.00

0.00

0.00

0.00

-..--

0.00

0.00

$\pm 0.43$
$-0.21$

0.00

$-4.45$

0.00

$-0.00$

$-0.00$

$-0.35$

0.00

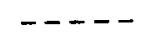

$-0.00$

0.00

$-5.01$
0.04 0.00

0.09

0.00

0.00

0.05

0.04

0.00

-..--

-....

0.00

0.00

$\pm 0.12$ 
Table 12.

SUMMARY OF THE SECULAR TIDAL ACCELERATION OF THE EARTH $\triangle$ A AND THE ACCELERATION OF THE MOON $i$ FROM THE GEM-TI OCEAN TIDAL MODEL

$\dot{\Omega}\left(10^{-22} \mathrm{rad} / \mathrm{sec}^{-2}\right)$

$\dot{\Omega}_{T, L P} \quad-0.17 \pm 0.14$

$\dot{\Omega}_{r, 0} \quad-0.80 \pm 0.12$

$\dot{\Omega}_{r, S D} \quad-5.01 \pm 0.12$

$\dot{\Omega}_{\mathrm{T}} \quad-5.98 \pm 0.22$

$\dot{\Omega}_{\mathrm{NT}} \quad+1.29 \pm 0.28$

$\dot{\Omega} \quad-4.69 \pm 0.36$ $\underline{\dot{n}\left(\operatorname{arcsec} c y^{-2}\right)}$

$\dot{n}_{T, L P} \quad-0.69 \pm 0.36$

$\dot{n}_{T, 0} \quad-3.18 \pm 0.25$

$\dot{n}_{T, S D} \quad-21.40 \pm 0.43$

$\dot{n}_{T} \quad-25.27 \pm 0.61$

in $\quad-25.27 \pm 0.61$ 


\section{BACK GROUND TIDES MODEL DEVELOPMENT}

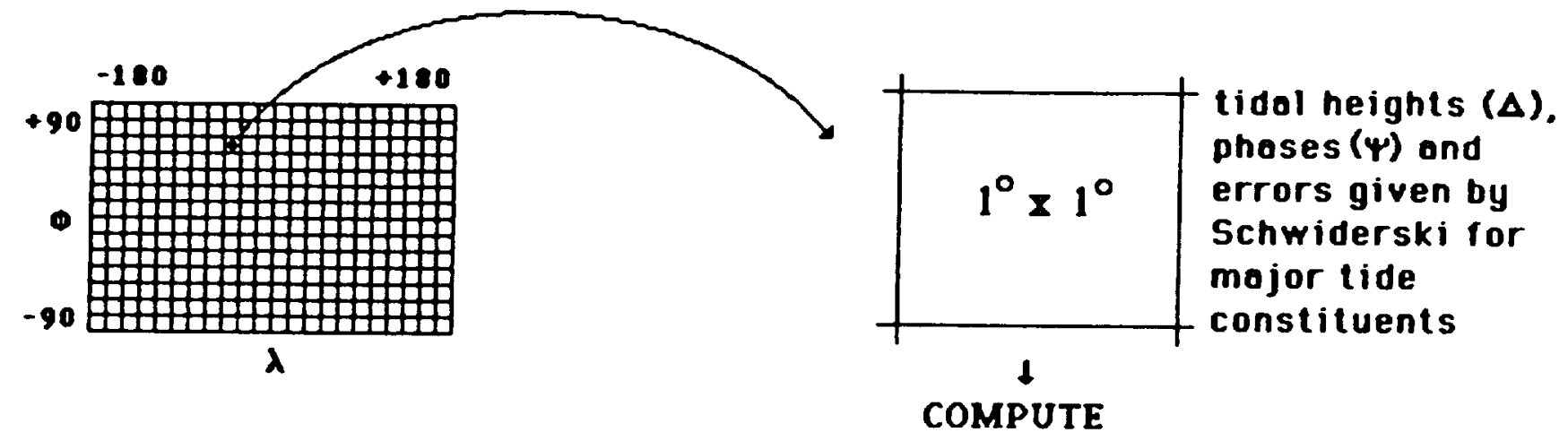

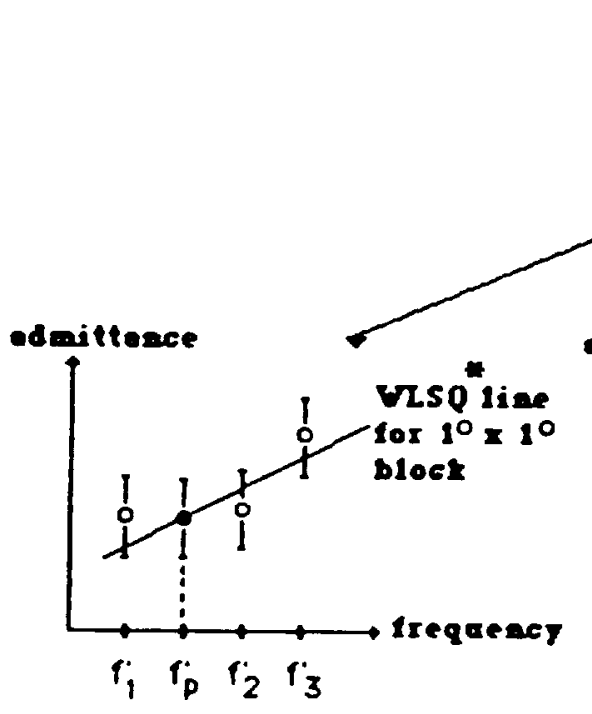

Long Period Band

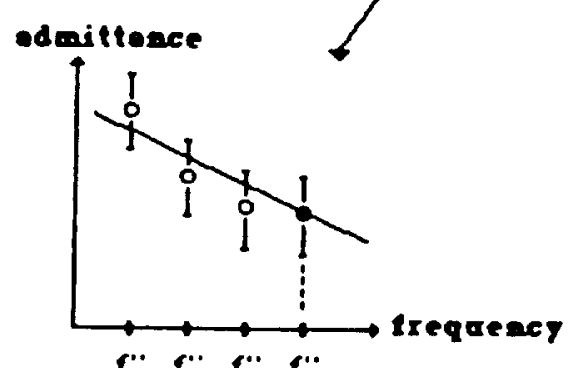

$f_{1} f_{2} f_{3} f_{p}$

Diurnal Band

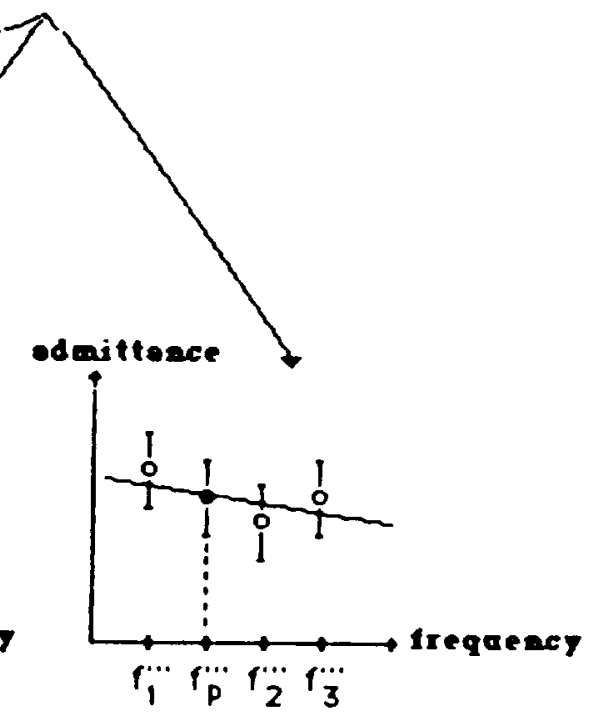

Semi-Diurnal Band

$$
\begin{aligned}
& \begin{array}{l}
i=\text { known admittances for } I_{1}, f_{2}, f_{3} \\
\downarrow \quad \begin{array}{c}
\text { (computed from Schwiderski } \\
\text { tides) }
\end{array}
\end{array} \\
& \text { i = predicted admittance for } I_{p} \text { 's } \\
& \text { I compute } \\
& \text { heights ( } \Delta \text { ), phases }(\Psi) \text { \& errors } \\
& \text { for } I_{p} \text { 's }
\end{aligned}
$$

FIGURE I 


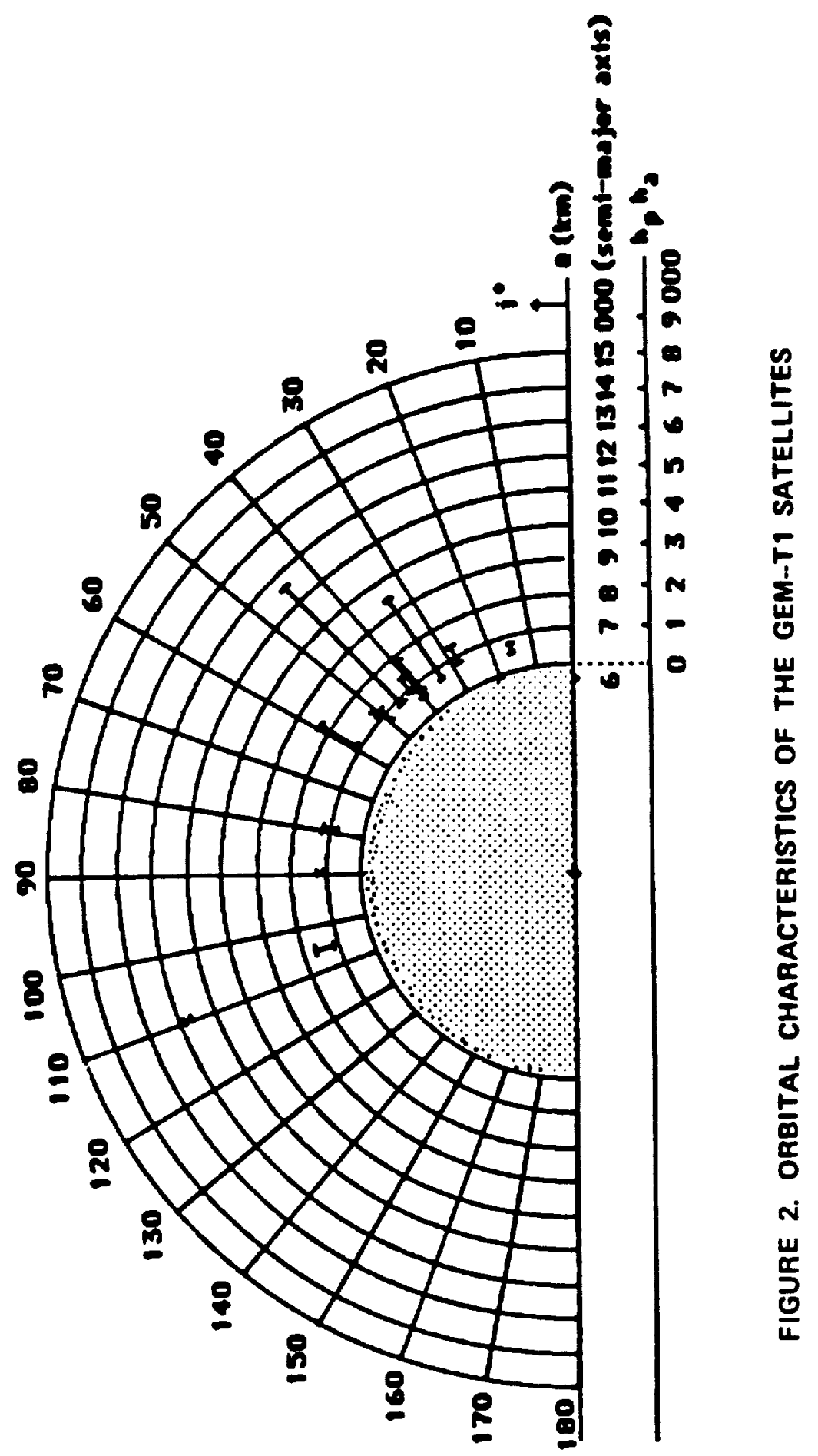




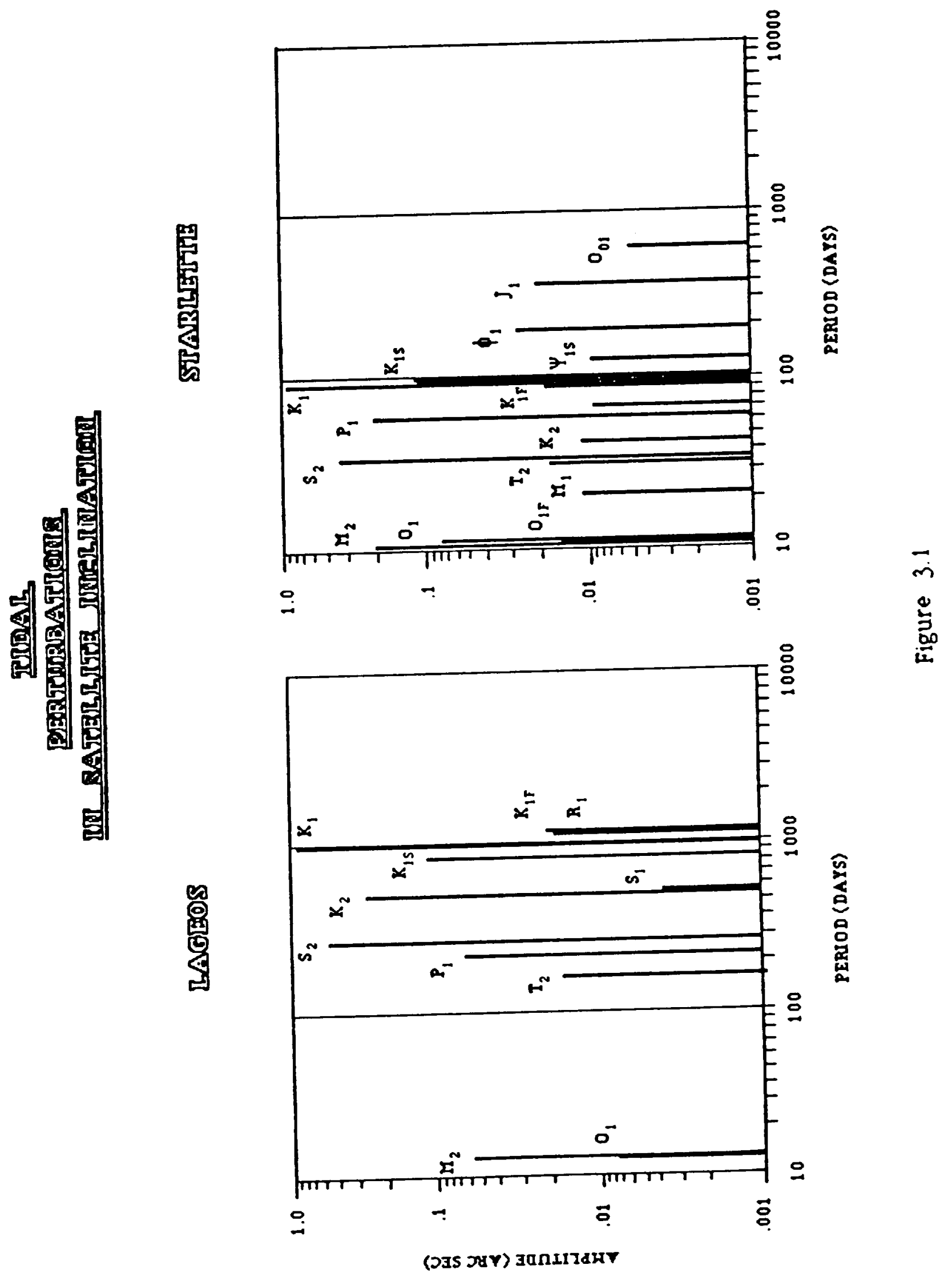



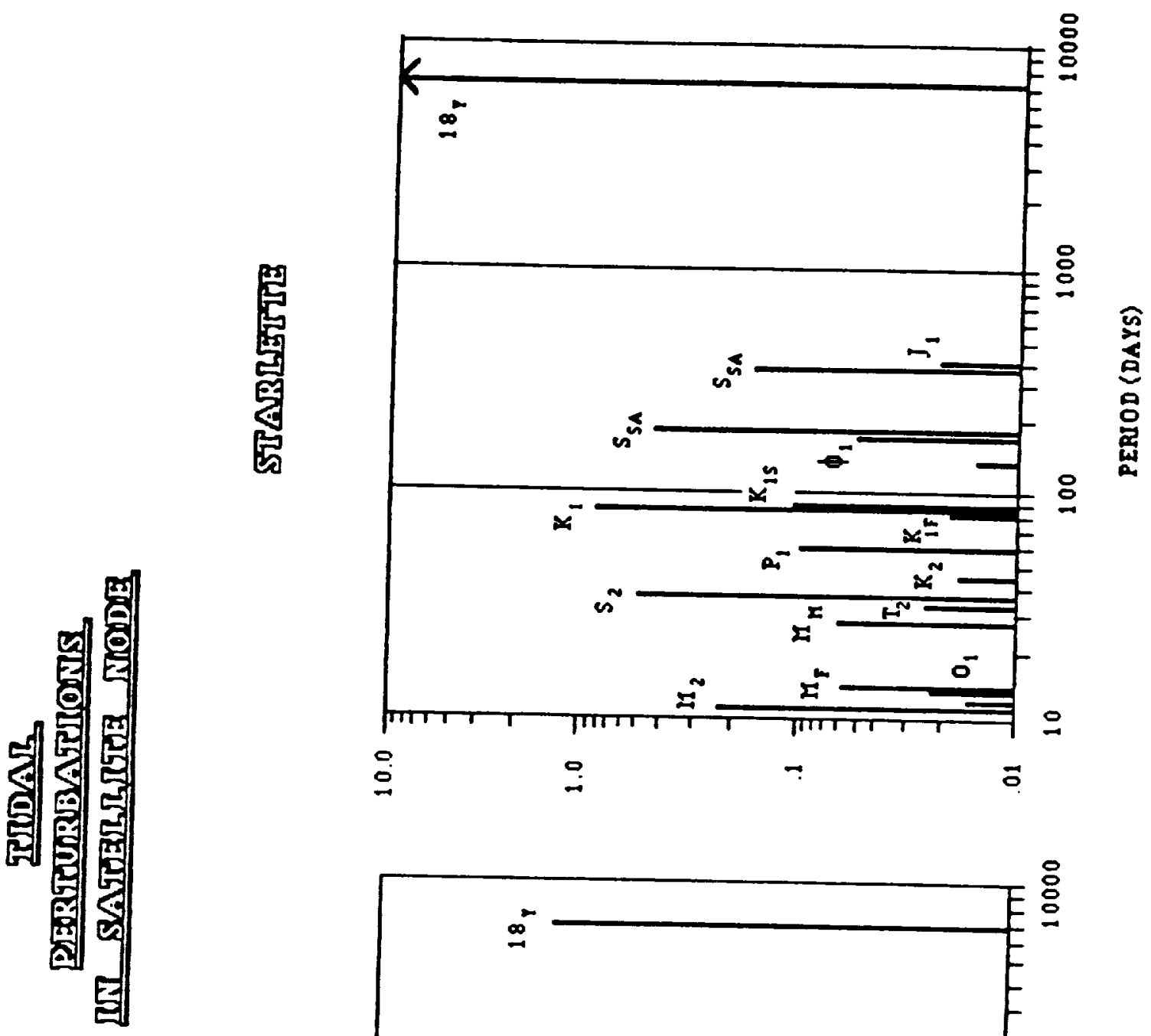

m

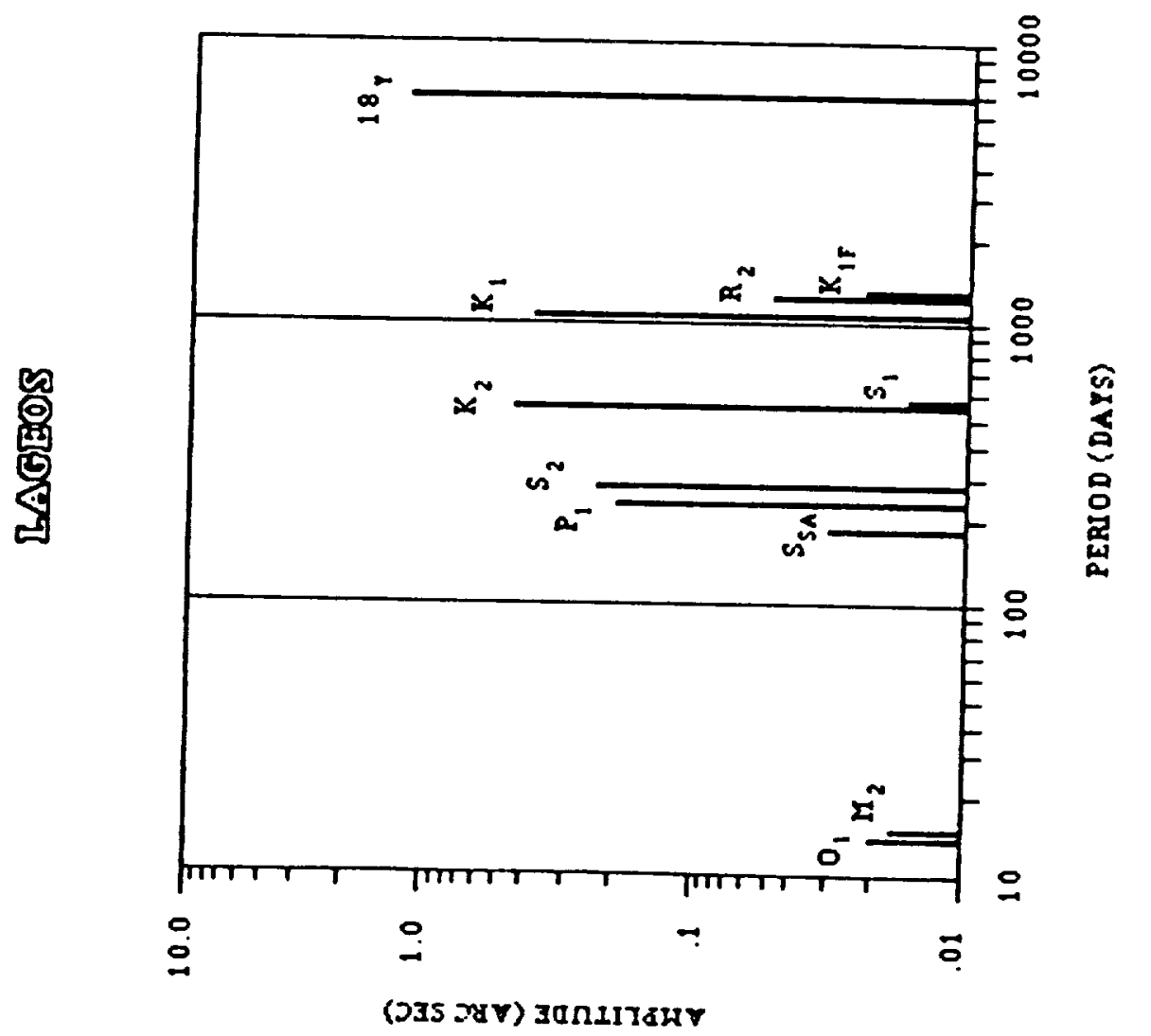




\section{PERIODS (DAYS) OF PRINCIPAL LONG PERIOD}

SATELLITE PERTURBRTIONS

DUE TO SOLID ERRTH RID OCERM TIDES

FOR 12 MAJOR TIDE CONSTITUENTS

\begin{tabular}{|c|c|c|c|c|c|c|c|c|c|c|c|c|}
\hline & $\begin{array}{l}n \\
\text { in } \\
\text { in } \\
0 \\
0 \\
0\end{array}$ & $\begin{array}{c}n \\
n \\
n \\
n \\
n \\
n \\
n \\
0\end{array}$ & $\begin{array}{l}n \\
n \\
n \\
8 \\
0 \\
0 \\
0 \\
0\end{array}$ & $\begin{array}{l}n \\
n \\
n \\
n \\
n \\
n \\
0 \\
0\end{array}$ & $\begin{array}{l}n \\
10 \\
18 \\
15 \\
0 \\
8 \\
0\end{array}$ & $\begin{array}{l}n \\
n \\
n \\
n \\
n \\
0\end{array}$ & $\begin{array}{l}n \\
n \\
n \\
n \\
n \\
0 \\
0\end{array}$ & 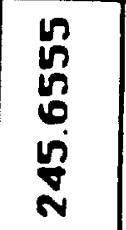 & 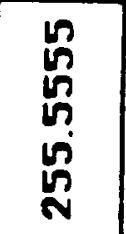 & $\begin{array}{l}n \\
0 \\
n \\
n \\
N \\
N \\
N\end{array}$ & $\begin{array}{l}n \\
n \\
n \\
n \\
n \\
n \\
N\end{array}$ & $\begin{array}{l}n \\
\text { in } \\
\text { in } \\
\text { in } \\
\text { n } \\
\underset{N}{N}\end{array}$ \\
\hline SATELLITE & s。 & $S_{80}$ & $M_{m}$ & $M_{\mathbf{f}}$ & $0_{1}$ & $P_{1}$ & $k_{1}$ & $\mathbf{N}_{2}$ & $M_{2}$ & $T_{2}$ & $\mathbf{s}_{\mathbf{2}}$ & $x_{2}$ \\
\hline LAGEOS & 365 & 183 & 27.6 & 13.7 & 13.8 & 221 & 1050 & 9.20 & 14.0 & 159 & 280 & 524 \\
\hline STARLETTE & 365 & 183 & 27.6 & 13.7 & 11.9 & 60.8 & 91.0 & 7.61 & 10.5 & 33.1 & 36.4 & 45.5 \\
\hline GEOS- 1 & 365 & 183 & 27.6 & 13.7 & 12.6 & 85.4 & 160 & 8.20 & 11.7 & 48.3 & 55.7 & 80.2 \\
\hline GEOS- 2 & 365 & 183 & 27.6 & 13.7 & 14.4 & 629 & 257 & 9.83 & 15.3 & 2250 & 436 & 129 \\
\hline GEOS-3 & 365 & 183 & 27.6 & 13.7 & 15.2 & 482 & 132 & 10.6 & 17.2 & 145 & 104 & 66.2 \\
\hline BE $-\mathbf{B}$ & 365 & 183 & 27.6 & 13.7 & 13.1 & 118 & 332 & 8.66 & 12.6 & 70.2 & 87.0 & 166 \\
\hline$B E-C$ & 365 & 183 & 27.6 & 13.7 & 11.8 & 57.9 & 84.8 & 7.51 & 10.3 & 31.5 & 34.4 & 42.4 \\
\hline SEASAT & 365 & 183 & 27.6 & 13.7 & 14.8 & 7130 & 178 & 10.2 & 16.1 & 331 & 174 & 89.0 \\
\hline TELSTAR- & 365 & 183 & 27.6 & 13.7 & 12.8 & 93.9 & 193 & 8.34 & 12.0 & 53.9 & 63.2 & 96.7 \\
\hline ANNA & 365 & 183 & 27.6 & 13.7 & 12.0 & 64.4 & 99.4 & 7.71 & 10.7 & 35.3 & 39.1 & 49.7 \\
\hline OSCAR & 365 & 183 & 27.6 & 13.7 & 13.6 & 180 & 11700 & 9.12 & 13.6 & 119 & 177 & 5830 \\
\hline
\end{tabular}

Figure 4 


\section{APPENDIX A \\ Mathematical Formulation of the \\ Earth and Ocean Tidal Potentials}

The purpose of this appendix is to provide the mathematical formulation of the solid earth and ocean tidal potentials appropriate to the use of osculating ephemerides. The relationship between the Kaula type indices and the equivalent Doodson numbering scheme is provided. Additionally, the linear orbit perturbations for the Kepler elements are given.

The formulation of the salid earth and ocean tidal potentials for use in Cowell type programs implies that the satellite accelerations are computed as the gradient of the potential:

$$
\left.\begin{array}{l}
\Gamma_{r}(\Phi, \lambda, r)=\frac{\partial u}{\partial r} \\
\Gamma_{\phi}(\Phi, \lambda, r)=\frac{1}{r} \frac{\partial U}{\partial \Phi} \\
\Gamma_{\lambda}(\Phi, \lambda, r)=\frac{1}{r \cos \Phi} \frac{\partial u}{\partial \lambda}
\end{array}\right\}
$$

In our sortware, GEODYN, the accelerations are evaluated in the inertial system aligned with the instantaneous earth-fixed system. These accelerations are then rotated to the inertial system of integration via transformations accounting for precession, nutation, polar motion and earth rotation.

These tidal potentials are formulated in terms of the ecliptic elements of the disturbing bodies and the equatorial elements of the satellite. The development we followed is quite similar to Goad (1977), wherein the surface harmonics due to the disturbing body are rotated from the equatorial to the ecliptic system. This formulation thus can use the planetary ephemerides (currently $D E-200$ ) directly, and the tidal perturbations are computed with the identical precise ephemeris used for other perturbations Using this form means that there are no terms which are dependant on 
both the sun and moon: these interaction effects are automatically generated by the numerical intergration process. It should also be noted that the distinction between mean and osculating is not currently significant ( $R$. J. Eanes, 1981, private communicotion)

\section{The Expressions Describing The Solid Earth And Ocean Tide Potentials}

The following initiol definitions are required

$$
\begin{aligned}
& \text { Ф. } \lambda . r \text { are the latitude, east langitude, ond redial } \\
& \text { distance of the point of evaluation, } \\
& \Theta_{\mathrm{g}} \quad \text { is the Greenwich sidereal hour angle, } \\
& P_{l q}(\cos J) \quad \text { are the associated Legendre functions } \\
& a^{*}, e^{*}, 1^{*}, \quad \text { ore the Keplerion elements of the disturbing } \\
& \Omega^{*}, \omega^{*}, M^{*} \text { body referred to the ecliptic, } \\
& a_{m}, e_{m}, i_{m} \quad \text { are the Keplerian elements of the moon referred } \\
& \Omega_{m}, \omega_{m}, M_{m} \text { to the ecliptic, } \\
& a_{s}, e_{s}, l_{s} \quad \text { are the Keplerian elements of the sun referred } \\
& \Omega_{s}, \omega_{s}, M_{s} \text { to the ecliptic, } \\
& \mu_{m} \quad \text { is the grovitational constant times the moss of } \\
& \text { the moon }
\end{aligned}
$$

$\mu_{s} \quad$ is the gravitational constant times the mass of the sun

is the gravitational constant times the mass of the earth

R is the average radius of the earth 
$G_{0}$

is the equivalent of the Doodson constant,

$\sum_{f}$

indicates summotion over all tide constituents (f's) in the expression of the tide genersting potential

$\bar{A}$

is the equivalent of the Doodson coefficient,

$k_{2, f}$

is the second degree love number and $\delta_{2, f}$

is its phase,

$k^{\prime}$

are the lood deformation coefficients,

$P_{0}$

is the average density of the oceanic water,

$C_{l a, f}^{ \pm}, \varepsilon_{l a, f}^{ \pm}$

are the amplitude and phase in the spherical

harmonic expansion of the ocean tides

specified by $l, q, \pm$, and the tide

constituent $f$

It can be shown that, in a system where the disturbing bodies (sun and moon) are referred to the ecliptic whereas the perturbed body is referred to the equator, the second degree tide potential is given by:

$$
V(\phi . \lambda . r)=\sum_{f} k_{2, f} \bar{A}_{f} G_{D} \frac{3-m}{3}\left(\frac{R}{r}\right)^{3} P_{2 m}(\sin \phi) \cos \alpha_{f}^{S E}
$$

for the solid earth, and by:

$U(\phi, \lambda, r)=\sum_{f} \sum_{l, q, \pm} 4 \pi G R \rho_{0}\left(\frac{1+k_{l}^{\prime}}{2 l+1}\right) C_{l q, f}^{ \pm}\left(\frac{R}{r}\right)^{l+1} P_{l q}(\sin \phi) \cos \alpha \frac{ \pm}{l q, f}$

for the oceans, where the angular arguments are respectively:

$$
\begin{gathered}
\alpha_{f}^{S E}=(\mp)\left[(2-2 h) \omega^{*}+(2-2 h+j) M^{*}+k \Omega^{*}\right]+m \Theta_{8}+m \lambda \\
+\pi-m^{\pi / 2}+\delta_{2, f}
\end{gathered}
$$

and 


$$
\begin{gathered}
\alpha_{l q, f}^{ \pm}=(\mp)\left[(2-2 h) \omega^{*}+(2-2 h+j) M^{*}+k \Omega^{*}\right]+m \Theta_{8} \pm q \lambda \\
+\pi-m \pi / 2+\varepsilon_{l q, f}^{ \pm}
\end{gathered}
$$

Note that there is a difference between \pm which belongs to the ocean tide expansion and $( \pm)$ which is part of the definition of the tidal constituent $f$. The tidal constituent $f$ corresponds to specification of $m, k, h, j,( \pm)$, and *. The equivalent of Doodson's constant is given by

$$
G_{D}=\frac{3}{4} \frac{\mu_{m} R^{2}}{8_{m}^{3}}
$$

The equivalent of the Doodson coefficient is given by

sun $\mid \bar{A}_{f}=\left\{\frac{\mu_{s}}{\mu_{m}}\left\{\frac{a_{m}}{a_{s}}\right\}^{3}\right\}(-1)^{\delta}\left(\frac{4}{3-m}\right) \frac{\left(2-\delta_{o m}\right)}{(2+m) !} \bar{\psi}_{2 m k}^{( \pm)} F_{2 k h}\left(i^{*}\right) G_{2 h j}\left(e^{*}\right)$

where

$$
\begin{aligned}
& \delta_{o m} \text { is the kronecker delta } \\
& d=\delta_{o k}+\frac{1}{2}[-k( \pm) k] \\
& \bar{\psi}_{2 m k}^{( \pm)}=\left(1-\frac{1}{2} \delta_{o k}\right) T_{2 m k}^{( \pm)}\left\{\frac{(2-k) !}{(2+k) !}\right\}_{(+)}^{(-)} \\
& T_{2 m k}^{( \pm)}=(\cos \tau)^{m( \pm) k}(\sin \tau)^{( \pm) k-m} F_{2 m k}^{( \pm)}\left(\cos ^{2} \tau\right) \\
& F_{2 m k}^{( \pm)}(x)=\frac{d^{2( \pm) k}}{d x^{2( \pm) k}}\left[x^{2-m}(x-1)^{2+m}\right] \\
& \tau=\frac{\varepsilon}{2} \text { ond } \varepsilon \text { is the obliquity of the ecliptic. }
\end{aligned}
$$


The inclination function $F_{2 k h}\left(i^{*}\right)$ and eccentricity function $G_{2 h j}\left(e^{*}\right)$ can be found in Kaule (1966, pp. 34-39).

The formulation of the potential in (1.2) and (1.3) is compatible with Doodson's development (Doodson, 1921). Doodson, however, elected to adopt Brown's lunar theory for the description of the position of the disturbing bodies, and thus his formulation is in terms of the mean elements of the sun and moon. In the present development, osculating elements are used.

\section{The Relationship To Doodson Agument Numbering}

It is often convenient to express the angular arguments in the same form as the Doodson solution. This requires the following osculating angular variables which correspond to Doodson's mean variables:

$\tau=$ local osculating luner hour angle, measured from lower transit of the moon past the local meridian:

$$
\tau=\alpha-s-\pi
$$

where $\alpha$ is the right ascension of the point of evaluation and $s$ is the moon's osculating longitude given by

$$
s=\Omega_{m}+\omega_{m}+M_{m}
$$

$P=$ moon's osculating longitude of perigee

$$
p=\Omega_{m}+\omega_{m}
$$

$N^{\prime}=$ the negative osculating longitude of the moon's ascending node

$$
N^{\prime}=-\Omega_{m}
$$

$h=$ the sun's osculating longitude

$$
n=\Omega_{5}+\omega_{s}+M_{5}
$$

$P_{1}=$ the osculating longitude of the sun's perigee

$$
p_{1}=\Omega_{s}+\omega_{s} \text {, and }
$$

$N_{1}^{\prime}=$ the negative osculating longitude of the sun's ascending node.

$$
N_{1}^{\prime}=-\Omega_{s}
$$


The angular argument of the tidal potential used by Doodson, allowing for the use of osculating variables, is:

$$
A_{\beta}=\beta_{1} \tau+\beta_{2} s+\beta_{3} h+\beta_{4} P+\beta_{5} N^{\prime}+\beta_{6} p_{1}+\beta_{7} N_{1}^{\prime}
$$

Where $\beta_{i}$ 's are integers which are typically in the range of -4 to 4 . As $\beta_{1}$ is always positive, the angular argument was expressed by Doodson as an "argument number":

$$
\beta_{1}\left(\beta_{2}+5\right)\left(\beta_{3}+5\right) \cdot\left(\beta_{4}+5\right)\left(\beta_{5}+5\right)\left(\beta_{6}+5\right)\left(\beta_{7}+5\right)
$$

Note that $\beta_{7}$ is not present in Doodson's development because the mean longitude of the sun's ascending node is by definition zero on the ecliptic

Using (1 19) and (1 12), the angular argument takes the form

$$
A_{\beta}=\beta_{1} \alpha+\left(\beta_{2}-\beta_{1}\right) s+\beta_{3} h+\beta_{4} P+\beta_{5} N^{\prime}+\beta_{6} P_{1}+\beta_{7} N_{1}-\beta_{1} \pi
$$

Ommission of the phase $\delta_{2, f}$ in equation (1.4) produces the angular argument of the tide generating potential which is equivalent to that expressed by equation (1.21) except for a possible offset. Allowing for the following definitions of the astronomical longitudes of the disturbing bodies

$$
\begin{aligned}
& L^{*}=\omega^{*}+M^{*}+\Omega^{*} \\
& P^{*}=\omega^{*}+\Omega^{*}
\end{aligned}
$$

the angular argument in (1.4) takes the form

$$
\alpha_{f}^{S E}-\delta_{2, f}=m \alpha+\pi-m \frac{\pi}{2}(\bar{f})\left[(2-2 h+j) L^{*}-j P^{*}-(2-2 h-k) \Omega^{*}\right]
$$

Note that $L^{*}$ is elther $s$ or $h, p^{*}$ is elther $p$ or $p_{1}$, and $\Omega *$ is elther $-N^{\prime}$ or $-N_{1}^{\prime}$. Also, the entire argument is either for the moon or for the sun, as mixed terms are not present in this formulation (Doodson has a small number of mixed terms which are present due to his choice of independent variables.)

By equating the coefficients of the independent variobles in (1.23) and (1.21), the following correspondences are obtained. 
a) Lunar Case

$$
\begin{array}{ll}
\beta_{1}=m & \beta_{5}=( \pm)(2-2 h-k) \\
\beta_{2}-\beta_{1}=(\mp)(2-2 h+j) & \beta_{6}=0 \\
\beta_{3}=0 & \beta_{7}=0 \\
\left.\beta_{4}=( \pm)\right\} &
\end{array}
$$

b) Solar Case

$$
\begin{array}{ll}
\beta_{1}=m & \beta_{5}=0 \\
\beta_{2}=\beta_{1}=m & \beta_{6}=( \pm) j \\
\beta_{3}=(\mp)(2-2 h+j) & \beta_{7}=( \pm)(2-2 h-k) \\
\beta_{4}=0 &
\end{array}
$$

The inverse correspondences are:

a) Lunar Case

$$
\begin{array}{rlrl}
\text { Let } \bar{k} & =\beta_{1}-\beta_{2}-\beta_{4}+\beta_{5} & \\
\text { For } \bar{k} \geq 0 & \jmath=\beta_{4} \\
m & =\beta_{1} & \text { sign }=(+) \\
n & =\left(\beta_{2}+\beta_{4}-\beta_{1}+2\right) / 2 & \\
k & =\bar{k} & & \\
\text { For } \bar{k} & <0 & & \text { sign }=(-) \\
m & =\beta_{1} & \\
n & =\left(\beta_{1}-\beta_{2}-\beta_{4}+2\right) / 2 & \\
k & =-\bar{k} &
\end{array}
$$


D) Solar cose

$$
\text { Let } \begin{array}{rlr}
\bar{k} & =\beta_{7}-\beta_{6}-\beta_{3} & \\
\text { For } \bar{k} & \geq 0 & \\
m & =\beta_{1} & \\
n & =\left(\beta_{3}+\beta_{6}+2\right) / 2 & \operatorname{sig} n=(+) \\
k & =\bar{k} &
\end{array}
$$

For $\vec{k}<0$

$$
\begin{array}{ll}
m=\beta_{1} & j=-\beta_{6} \\
n=\left(-\beta_{3}-\beta_{6}+2\right) / 2 & \text { sign }=(-) \\
k=-\bar{k} &
\end{array}
$$

Note that when either $k$ or $m$ is zero, there is a twofold symmetry in that twa different choices of indices generate the sarre frequencies when both $m$ and $k$ are zero, a fourfold symmetry exists. This is so whether the Doodson notation or the kaula type indices are used

The Doodsan coefficients indicated by (1.7) must be surrimed aver the symmetric cases to compare with Doodson's tables, in these cases the vaiues of the separate coefficients are identical Additianally, when the angular argument is independent of the elements af the disturbing body $(h=1, j=0, k=0)$, each disturbing body has a twofold symmetry and the coefficients must also be summed over the disturbing bodies (i.e, for the permanent tide, $k_{1}$, srid $k_{2}$ ) 
The analytical formulations for the tidal perturbations of the Kepler elements of a satellite are derived below. As can be seen from inspection of equations (1.2), (1.3), (1.4) and (1.5), the solid earth potential is a special case of the ocean tide potential Thus the following derivations are for the ocean potential, and the solid earth case will be derived therefrom

Let

$$
\begin{aligned}
& K_{l q, f}^{ \pm}=\frac{4 \pi G R^{2} \rho_{0}}{\mu}\left(\frac{1+k_{l}^{\prime}}{2 l+1}\right) C_{l q, f}^{ \pm} \\
& \sigma_{l q, f}^{ \pm}=\alpha_{l q, f}^{ \pm} \mp q \lambda \text {, and } \\
& \mu=\text { the gravitational constant times the mass of } \\
& \quad \text { the earth }
\end{aligned}
$$

The tide potential given in (1.2) for the oceans can then be rewritten as:

$$
U_{f}(\phi, \lambda, r)=\sum_{\ell, q, \pm} K_{\ell q, f}^{ \pm} \frac{\mu R^{\ell}}{r^{l+1}} P_{l q}(\sin \phi) \cos \left(\sigma_{l q, f}^{ \pm} \pm q \lambda\right)
$$


Letting

$$
\begin{aligned}
& A_{l q, f}=K_{l q, f}^{+} \cos \sigma_{l q, f}^{+}+K_{l q, f}^{-} \cos \sigma_{l q, f}^{-} \\
& B_{l q, f}=-K_{l q, f}^{+} \sin \sigma_{l q, f}^{+}+K_{l q, f}^{-} \sin \sigma_{l q, f}^{-}
\end{aligned}
$$

a particular $l, q$ term of the potential can be written as

$$
U_{l q, f}=\frac{\mu R^{l}}{r_{l+1}} P_{l q}(\sin \phi)\left[A_{l q, f} \cos q \lambda+B_{l q, f} \sin q \lambda\right]
$$

which is of the same form as the potential given by Koulo (1966, p. 31, eq $(3,53))$ Carrying on in the same fashion as Kaula did, $(130)$ takes the form (see ibid ea $3,70 \& 3,71$ )

$$
U_{l q, f}=\frac{\mu R^{l}}{g^{l+1}} \sum_{p=0}^{l} F_{l q p}(i) \sum_{s=-\infty}^{\infty} G_{l p s}(e) S_{l q p s, f}\left(\omega, M, \Omega, \theta_{8}\right)
$$

where $\theta, e, 1, \omega, M, \Omega$ are the Kepler elements of the perturbed body, the functions $F_{Q q p}$ and $G_{\text {Qps }}$ are given in Koulo (1966; pp. 34-38), and

$$
S_{l \text { qps }, f}\left(\omega, M, \Omega, \theta_{8}\right)=\left[\begin{array}{c}
A_{l q}, f \\
-B_{l q, f}
\end{array}\right]_{l-q \text { odd }}^{l-q \text { even }} \cos x_{l q p s}+\left[\begin{array}{l}
B_{l q, f} \\
A_{l q}, f
\end{array}\right]_{l-q \text { odd }}^{l-q \text { even }} \sin x_{l q p s}
$$

with

$$
x_{l q p s}=(l-2 p) \omega+(l-2 p+s) M+q\left(\Omega-\theta_{8}\right)
$$

It can be easily shown that $S_{\text {gqps if }}$ may also be written as

$$
S_{l q p s, f}\left(W, M, \Omega, \theta_{8}\right)=\sum_{+} K_{l q, f}^{ \pm}\left[\begin{array}{c}
\cos \\
\sin
\end{array}\right]_{l-q \text { add }}^{l-q \text { even }}\left(x_{l q p s} \pm \sigma_{l q, f}^{ \pm}\right)
$$


The time rate of change of the argument

$$
\gamma_{\text {eqps,f }}^{ \pm}=x_{\text {eqps }} \pm \sigma_{\text {eq,f }}^{ \pm}
$$

is dominated by the equatorial secular rates in $\omega, \Omega$, and $M$ of the sotellite, by the rotation of the earth represented by $\theta_{8}$. and by the ecliptic secular rates of the disturbing body in $\omega^{*}, \Omega^{*}$, and $M^{*}$. Hence $\ddot{\gamma}_{\ell q p s, f}$ is opproximetely constent over the period associated with $S_{\rho q p s, f}$. The linear perturbations in the orbit elements can be constructed (Koula, 1966, pp. $37-41$ ) for each term desired by analytically integrating the Lagrangian equations of motion for that term:

$$
\begin{aligned}
& \Delta \theta_{l q p s, f}=\mu R^{l} \frac{2 F_{l q p} G_{l p s}(l-2 D+9)}{n \theta^{l+2} \dot{\gamma}_{l q p s, f}} S_{l q p s, f} \\
& \Delta e_{l q p s, f}=\mu R^{l} \frac{F_{l q p} G_{l p s}\left(1-e^{2}\right)^{\frac{1}{2}}\left[\left(1-e^{2}\right)^{\frac{1}{2}}(l-2 p+s)-(l-2 D)\right]}{n \theta^{l+3} \mathrm{e} \dot{\gamma}_{l q p s, f}} S_{l q p s, f} \\
& \Delta i_{l q p s, f}=\mu R^{l} \frac{F_{l q p} G_{l p s}[(l-2 p) \cos i-q]}{n \theta^{l+3}\left(1-e^{2}\right)^{\frac{1}{2}} \sin 1 \dot{\gamma}_{l q p s, f}^{ \pm}} S_{l q p s, f}
\end{aligned}
$$

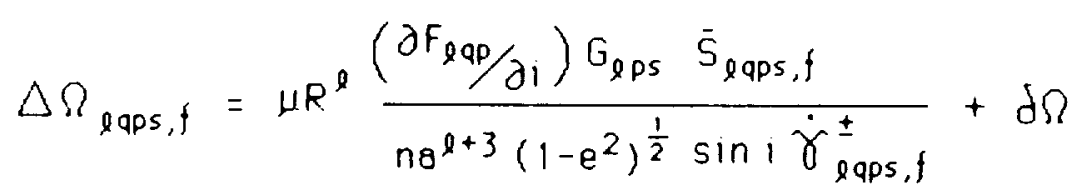

$$
\begin{aligned}
& \left.\Delta \omega_{l q p s, f}=\mu R^{l}\left[\left(1-e^{2}\right)^{\frac{1}{2}} e^{-1} F_{l q p}\left(\partial G_{Q p s} / \partial e\right)-\operatorname{coti}\left(1-e^{2}\right)^{-\frac{1}{2}\left(\partial F_{l q p} / \partial i\right.}\right) G_{l p s}\right] \\
& n \theta^{l+3} \quad \dot{\gamma}_{l q p s, f}^{ \pm} \\
& +\delta \omega \\
& \Delta M_{\ell q p s, f}=\frac{\mu R^{l}\left[-\left(1-e^{2}\right) e^{-1}\left(\partial G_{l p s} / \partial e\right)+2(l+l) G_{l p s}-3 n G_{l p s}(l-2 p+s)\left(\dot{\gamma} \pm \frac{t}{l q p s, f}\right)^{-1}\right]}{n \theta^{l+3} \dot{\gamma}_{l q p s, f}^{ \pm}}
\end{aligned}
$$


where $n$ is the mean motion of perturbed body:

$$
n=\sqrt{\frac{\mu}{a^{3}}} \text {, }
$$

$\bar{S}_{\text {lqps,f }}$ is the integral of $S_{\text {lqps,f }}$ with respect to its argument:

$$
\vec{S}_{l q p s, f}=\sum_{+} K_{l q, f}^{ \pm}\left[\begin{array}{r}
\sin \\
-\cos
\end{array}\right]_{l-q \text { odd }}^{l-q \text { even }} \gamma_{l q p s, f}^{ \pm}
$$

and $\delta \Omega, \delta \omega, \delta M$ are the indirect effects due to the earth's oblateness. From Bolmino (1974):

$$
\begin{gathered}
{\left[\begin{array}{l}
\delta \Omega \\
\delta \omega \\
\delta M
\end{array}\right]=3 n C_{20}\left(\frac{R}{\theta}\right)^{2}\left[\begin{array}{l}
\frac{1}{2\left(1-e^{2}\right)^{2}} \\
\frac{1}{\left(1-e^{2}\right)^{2}} \\
\frac{3}{4\left(1-e^{2}\right)^{3 / 2}}
\end{array}\right]\left\{\frac{e}{\left(1-e^{2}\right)}\left[\begin{array}{l}
4 \cos i \\
1-5 \cos ^{2} i \\
1-3 \cos ^{2} i
\end{array}\right] E\right.} \\
\left.+\left[\begin{array}{l}
-\sin i \\
\frac{5}{4} \sin 2 i \\
\sin 2 i
\end{array}\right] I\right\}
\end{gathered}
$$

with the earth's second degree harmonic $C_{20} \approx-1.082628 * 10^{-3}$ and

$$
\begin{aligned}
& E=\mid \Delta e_{\text {lqps }, f}(t) \\
& I=\mid \Delta i_{\text {lqps }, f}(t)
\end{aligned}
$$

Integrating the inclination, we get

$$
I=\mu R^{l} \frac{F_{l q p} G_{l p s}[(l-2 p) \cos i-q]}{n \theta^{l+3}\left(1-e^{2}\right)^{\frac{l}{2}} \sin i\left(\dot{\gamma}_{l q p s, f}^{t}\right)^{2}} \bar{S}_{l q p s, f}
$$


and for the eccentricity

$$
E=\mu R^{l} \frac{F_{l q p} G_{l p s}\left(1-e^{2}\right)^{\frac{l}{2}}\left[\left(1-e^{2}\right)^{\frac{l}{2}}(l-2 p+s)-(l-2 p)\right]}{n \theta^{l+3} e\left(\dot{\gamma}_{l q p s, f}^{ \pm}\right)^{2}} \bar{S}_{l q p s, f}
$$

Substituting $(1.48)$ and $(1.49)$ to $(1.46)$ and by letting

$$
S_{l q p s, f}=\frac{4 \pi G R^{2} \rho_{0}}{\mu}\left(\frac{1+K_{l}}{2 l+1}\right) Q_{l q p s, f}
$$

and

$$
\bar{S}_{\text {laps }, f}=\frac{4 \pi G R^{2} \rho_{0}}{\mu}\left(\frac{1+k_{l}^{\prime}}{2 l+1}\right) \bar{Q}_{l \text { qps }, f}
$$

where

$$
\begin{aligned}
& \mathrm{Q}_{\ell \text { qps }, f}=\bar{\sum}_{+} C_{l q, f}^{ \pm}\left[\begin{array}{l}
\cos \\
\sin
\end{array}\right]_{\ell \text {-q odd } \gamma_{l q p s, f}^{l-q \text { even }}}^{ \pm} \\
& \bar{Q}_{\ell q p s, f}=\bar{\sum}_{+} C_{l q, f}^{ \pm}\left[\begin{array}{l}
\sin \\
-\cos
\end{array}\right]_{l \text {-q odd } \gamma_{l q p s, f}^{l-q \text { even }}}^{ \pm}
\end{aligned}
$$

we have

$$
\begin{gathered}
\delta \Omega=\frac{6 \pi G \rho_{0} C_{20}}{\left(1-e^{2}\right)^{5 / 2} \theta}\left(\frac{R}{\theta}\right)^{l+4}\left(\frac{1+k_{l}^{\prime}}{2 l+1}\right) \frac{F_{l q p} G_{l p s}}{\left(\dot{\gamma}_{l q p s, f}^{ \pm}\right)^{2}} \bar{Q}_{l q p s, f} \\
\cdot\left[q+4 \cos 1\left(1-e^{2}\right)^{l / 2}(l-2 p+s)-5 \cos i(l-2 p)\right] \\
\delta \omega=\frac{6 \pi G p_{0} C_{20}}{\left(1-e^{2}\right)^{5 / 2} \theta}\left(\frac{R}{8}\right)^{l+4}\left(\frac{1+k_{l}^{\prime}}{2 l+1}\right) \frac{F_{l q p} G_{l p s}}{\left(\dot{\gamma}_{l q p s, f}^{ \pm}\right)^{2}} \bar{Q}_{l q p s, f} \\
\cdot\left[15 \cos ^{2} i(l-2 p)-5 q \cos 1+2\left(1-5 \cos ^{2} i\right)\left(1-e^{2}\right)^{l / 2}(l-2 p+s)-2(l-2 p)\right]
\end{gathered}
$$




$$
\begin{aligned}
& \delta M=\frac{9 \pi G \rho_{0} C_{20}}{\left(1-e^{2}\right)^{2} \theta}\left(\frac{R}{a}\right)^{l+4}\left(\frac{1+K_{l}^{\prime}}{2 l+1}\right) \frac{F_{l q p} G_{l p s}}{\left(\dot{\gamma}_{l q p s, f}^{ \pm}\right)^{2}} \bar{Q}_{l q p s, f} \\
& \cdot\left[5 \cos ^{2} i(l-2 p)-2 q \cos i-(l-2 p)+\left(1-3 \cos ^{2} i\right)\left(1-e^{2}\right)^{\prime 2}(l-2 p+s)\right]
\end{aligned}
$$

From (1.38) through (1.56) we con write the final form of the perturbation in the Kepler elements due to the ocesnic tidal potential:

$$
\Delta a_{l q p s, f}=\frac{8 \pi G p_{0}}{n \dot{\gamma}_{l q p s, f}^{ \pm}}\left(\frac{R}{\theta}\right)^{l+2}\left(\frac{1+k_{\ell}}{2 l+1}\right) F_{l q p} G_{l p s}(l-2 p+s) Q_{l q p s, f}
$$

$$
\begin{aligned}
\Delta e_{Q q p s, f}=\frac{4 \pi G \rho_{0}\left(1-e^{2}\right)^{\frac{1}{2}}}{\text { nae } \dot{\gamma}_{l q p s, f}^{ \pm}} & \left(\frac{R}{8}\right)^{l+2}\left(\frac{1+k_{l}^{\prime}}{2 l+1}\right) F_{l q p} G_{l p s} \\
\cdot & {\left[\left(1-e^{2}\right)^{\frac{1}{2}}(l-2 p+s)-(l-2 p)\right] Q_{l q p s, f} }
\end{aligned}
$$

$$
\begin{aligned}
\Delta i_{l q p s, f}= & \frac{4 \pi G p_{0}}{n a\left(1-e^{2}\right)^{\prime 2} \sin i \dot{\gamma}_{l q p s, f}^{ \pm}}\left(\frac{R}{\theta}\right)^{l+2}\left(\frac{l+k_{l}^{\prime}}{2 l+l}\right) F_{l q p} G_{l p s} \\
& {[(l-2 p) \cos i-q] Q_{l q p s, f} }
\end{aligned}
$$




$$
\begin{aligned}
& \Delta \Omega_{l q p s, f}=\frac{4 \pi G \rho_{0} G_{l p s} \bar{Q}_{l q p s, f}}{n \theta\left(1-e^{2}\right)^{\frac{1}{2}} \sin 1 \dot{\gamma}_{l q p s, f}^{ \pm}}\left(\frac{R}{\theta}\right)^{l+2}\left(\frac{1+k_{l}^{\prime}}{2 l+1}\right) \\
& \text { - }\left[\left(\frac{\partial F_{\ell q \rho}}{\partial i}\right)-\frac{3}{2} \frac{C_{20} n \sin i F_{l q p}}{\left(1-e^{2}\right)^{2} \dot{\gamma}_{l q p s}^{ \pm} f}\left(\frac{R}{\theta}\right)^{2}\left(5 \cos i(l-2 p)-4 \cos i\left(1-e^{2}\right)^{1 / 2}\right.\right. \\
& (l-2 p+s)-q)] \\
& \Delta \omega_{l q p s, f}=\frac{4 \pi G \rho_{0} \bar{Q}_{l q p s, f}}{n a\left(1-e^{2}\right)^{\prime 2} \dot{\gamma}_{l q p s, f}^{ \pm}}\left(\frac{R}{8}\right)^{l+2}\left(\frac{1+k_{l}^{\prime}}{2 l+1}\right) \\
& \text { - }\left[\frac{1-e^{2}}{e}\left(\frac{\partial G_{l p s}}{\partial e}\right) F_{l q p}-\cot i\left(\frac{\partial F_{l q p}}{\partial i}\right) G_{l p s}+\frac{15}{2} \frac{C_{20} n F_{l q p} G_{l p s}}{8^{2}\left(1-e^{2}\right)^{2} \dot{\gamma}_{l q p s, f}^{ \pm}}\left(\frac{R}{\theta}\right)^{2}\right. \\
& \left.\cdot\left[3 \cos ^{2} i(l-2 p)-q \cos 1+\frac{2}{5}\left(1-5 \cos ^{2} i\right)\left(1-e^{2}\right)^{\frac{1}{2}}(l-2 p+s)-\frac{2}{5}(l-2 p)\right]\right]
\end{aligned}
$$

$$
\begin{aligned}
& \Delta M_{l q p s, f}=\frac{4 \pi G_{\rho_{0}} F_{l q p} \bar{Q}_{l q p s, f}}{n \theta \dot{\gamma}_{l q p s, f}^{ \pm}}\left(\frac{R}{a}\right)^{l+2}\left(\frac{1+k_{l}^{\prime}}{2 l+1}\right)\left[-\frac{1-e^{2}}{e}\left(\frac{\partial G_{l p s}}{\partial e}\right)\right. \\
& +2(l+1) G_{l p s}-\frac{3 n G_{l p s}(l-2 p+s)}{\dot{\gamma}_{l q p s, f}^{ \pm}}+\frac{9}{4} \frac{C_{20} n G_{l p s}}{\left(1-e^{2}\right)^{2} \dot{\gamma}_{l q p s, f}^{ \pm}}\left(\frac{R}{\theta}\right)^{2}\left[5 \cos ^{2},(l-2 p)\right. \\
& \left.-2 q \cos 1-(l-2 p)+\left(1-3 \cos ^{2} i\right)\left(1-e^{2}\right)^{\left.\frac{1}{2}(l-2 p+s)\right]}\right]
\end{aligned}
$$


Perturbation amplitudes given by (1.57)..(1.62) can also be expressed in the form of position perturbations in the radial $(\Delta r)$, transverse $(\Delta t)$, and normal $(\Delta n)$ directions (Williamson and Douglas, 1972):

$$
\begin{aligned}
& \Delta t=a\left(1+\frac{1}{2} e^{2}\right)^{\frac{1}{2}}\left(\Delta M+\Delta \omega+\Delta \Omega \cos i+\left(\Delta e^{2}+(e \Delta M)^{2}\right)^{1 / 2}\right) \\
& \Delta r=\left\{\Delta \theta^{2}+\frac{1}{2}\left[(e \Delta a+a \Delta e)^{2}+(a e \Delta M)^{2}\right]\right\}^{1 / 2} \\
& \Delta n=g\left(1+\frac{1}{2} e^{2}\right)^{1 / 2}\left(\frac{1}{2} \Delta i^{2}+\Delta \Omega^{2} \sin ^{2} 1\right)^{1 / 2}
\end{aligned}
$$

and for the velocity perturbations

$$
\begin{aligned}
\Delta Y_{t} & =\left\{\left(\frac{\Delta \theta}{2 a^{3 / 2}}\right)^{2}+\frac{1}{2}\left[\left(\frac{\Delta e}{\sqrt{\theta}}-\frac{\Delta a e}{2 \theta^{3 / 2}}\right)^{2}+\left(\frac{e}{\sqrt{\theta}} \Delta M\right)^{2}\right]\right. \\
+ & {\left.\left[\theta\left(1+\frac{1}{2} e^{2}\right)^{1 / 2} \cos 1 \frac{d \Omega}{d t}\right]^{2}\right\}^{1 / 2} } \\
\Delta Y_{r} & =\frac{\sqrt{2}}{2}\left[\left(\frac{\Delta e}{\sqrt{a}}-\frac{e \Delta \theta}{2 a^{3 / 2}}\right)^{2}+\left(\frac{e}{\sqrt{g}} \Delta M\right)^{2}\right]^{1 / 2}
\end{aligned}
$$

and,

$$
\Delta V_{n}=8\left(1+\frac{1}{2} e^{2}\right)^{1 / 2}\left\{\frac{1}{2}\left[\left(\frac{d i}{d t}\right)^{2}+n^{2} \Delta 1^{2}\right]+\left(\frac{d \Omega}{d t}\right)^{2} \sin ^{2} i\right\}^{1 / 2}
$$

For formulas (1.63) through (1.68). we note the perturbation amplitudes are the RSS values of the perturbations and that small eccentricity approximations have been extensively applied 
Formulas for the perturbations due to the solid earth tides can be obtained from (1.52) (1.57) by making the following substitutions

OCEAN TIDES

Replace
$4 \pi G \rho_{0} R\left(\frac{1+k_{y}^{\prime}}{2 y+1}\right) C_{l 2, f}^{ \pm}$

l

7

$\pm$

$\varepsilon_{l a, t}^{ \pm}$
SOLID EARTH

With

$$
k_{2, f} \bar{A}_{f} G_{D}\left(\frac{3-m}{3}\right)
$$

2

m

$+$

$\delta_{2, f}$

From formulas (1.57) through (1.62) one can derive principal long-period perturbations for ocean or solid earth tides when

$$
P=l / 2, \quad s=0, \quad+\operatorname{sign} \text { and } q=m
$$

Then the angular argument becomes

$$
\gamma_{l m^{l} / 2, f}^{+}=(F)\left[(2-2 h) \omega^{*}+(2-2 h+j) M^{*}+k \Omega^{*}\right]+m \Omega+\pi-m \frac{\pi}{2}+\varepsilon_{l m, f}^{+}
$$

with frequencies given by

$$
\dot{\gamma}_{l m^{l} / 20, f}^{+}=(\mp)\left[(2-2 h) \dot{\omega}^{*}+(2-2 h+j) \dot{M}^{*}+k \dot{\Omega}^{*}\right]+m \dot{\Omega}
$$

It is apparent from (1.71) that the principal perturbations due to the solid earth and ocean tides have the same periods and therefore cannot be distinguished. The satellite senses the total tidal effect 



\section{MATHEMATICAL FORMULATION OF THE SECULAR CHANGES IN THE LUNAR AND SOLAR ORBITS}

Due to the anelastic behavior of the Earth's response and the dissipation of energy in the oceans, the earth's tidal bulge causes secular changes in the motion of the moon and the sun. The moon's orbit and the sun's apparent orbit can be treated in a way similar to that described in Appendix 1 and analytical perturbations in their orbital elements can be obtained from equations (157) through (162). It is evident, however, that secular perturbations will be generated only when the angular arguments given by equations (14) and (1.5) are independent of time Necessary conditions for the cancellation of the angular elements of the sun and the moon are that the motions of both the disturbing and the perturbed bodies, which are the same in this case, be referred to the same plane The mathematical formulation of the tidal potential given in Appendix 1 , is in a system where the disturbing bodies are referred to the ecliptic whereas the perturbed body is referred to the equator. The formulation of the potential, where the eclidtic is the reference frome common to both bodies but the tide amplitude and phase are in the usual equatorial reference frame, is given herein. The formulation of the potential is developed for the case of the oceans only since the solid earth is a special case of this development.

From equation (15), we can write:

$$
\alpha_{l q, f}^{ \pm}=\vartheta_{ \pm q \lambda}
$$

where

$$
\begin{aligned}
\vartheta= & (\mp)\left[(2-2 h) \omega^{*}+(2-2 h+\jmath) M^{*}+k \Omega^{*}\right] \\
& +m \Theta_{8}-\frac{m \pi}{2}+\pi+\varepsilon_{\ell q, f}^{ \pm}
\end{aligned}
$$


Letting

$$
\Lambda_{l q, f}^{ \pm}=4 \pi G R_{\rho_{0}}\left(\frac{1+k_{l}^{\prime}}{2 l+1}\right) C_{l q, f}^{ \pm}
$$

arid noting that

$$
\cos (\Im( \pm) q \lambda)=\cos (q \lambda( \pm) \vartheta)
$$

the potential given by (1.3) can be rewritten as:

$$
U_{l q, f}^{ \pm}=\Lambda_{l q, f}^{ \pm}\left(\frac{R}{r}\right)^{l+1} P_{l q}(\sin \phi) \cos (q \lambda( \pm) \&)
$$

which is the real port of the complex potential

$$
U_{l q, f}^{ \pm}=\Lambda_{l q, f}^{ \pm}\left(\frac{R}{r}\right)^{l+1} P_{l q}(\sin \theta) e^{i(q \lambda( \pm) \vartheta)}
$$

where

$$
i=\sqrt{-1}
$$

Substituting the right ascension $(\alpha)$ for the longltude $(\lambda)$ in equation (26), gives

$$
U_{g q, f}^{ \pm}=A_{g Q, f}^{ \pm} e^{\left(1-q 0_{8}( \pm) g\right)}\left(\left(\frac{R}{r}\right)^{l+1} P_{l q}(\sin \theta) e^{1 q \alpha}\right)
$$

Using the approsch of Good (1977),

$$
\begin{aligned}
\left(\frac{R}{r}\right)^{l+1} P_{l q}(\sin \theta) e^{j q \alpha} & =\left(\frac{R}{r}\right)^{l+1} \frac{1}{(l-q)} \sum_{v=0}^{\ell} \sum_{\{ \pm\}} \psi_{l q v}^{\{ \pm\}} \\
& \cdot P_{l v}\left(\sin \theta^{*}\right) e^{i\left[\left\{t r \alpha^{*}-(v-q) \pi / 2\right]\right.}
\end{aligned}
$$


where the starred elements refer to the ecliptic and the function $\psi_{g q v}^{( \pm+\}}$ is given in equation (19) of Appendix 1. Substituting (2.8) to (2.7) gives

$$
\begin{aligned}
& U_{l q, f}^{ \pm}=n_{l q, f}^{ \pm} \frac{1}{(l-q) !} \sum_{v=0}^{l} \sum_{\{ \pm\}} e^{i\left(-q \theta_{g}( \pm) \vartheta+(q-v) \frac{\pi}{2}\right)} \\
& \cdot \psi_{q q \vee}^{\{ \pm\}} \cdot\left\{\left(\frac{R}{r}\right)^{l+1} P_{l v}\left(\sin \phi^{*}\right) e^{i\left(\{ \pm\} \vee \alpha^{*}\right)}\right\}
\end{aligned}
$$

From Kaula (1966)

$$
\left(\frac{R}{r}\right)^{l+1} P_{Q v}\left(\sin 0^{*}\right) e^{i v \alpha^{*}}=\left(\frac{R}{\theta^{*}}\right)^{l+1} \sum_{p=0}^{l} \sum_{s=-\infty}^{\infty} F_{l v p}(i *) G_{l p s}\left(e^{*}\right) e^{j \tau_{l v p s}^{*}}
$$

where

$$
\tau_{Q v p s}^{*}=(l-2 p) \omega^{*}+(l-2 p+s) M^{*}+v \Omega^{*}-\frac{\pi}{2} \bmod (l-v, 2)
$$

and therefore (2.9) becomes

$$
\begin{aligned}
& U_{l q, f}^{ \pm}=\Lambda_{q q, f}^{ \pm} \frac{1}{(l-q) !} \sum_{\gamma, p, s} e^{i\left(-q \theta_{g}( \pm) \vartheta+(q-v) \frac{\pi}{2}\right)}\left(\frac{R}{a^{*}}\right)^{l+1}
\end{aligned}
$$

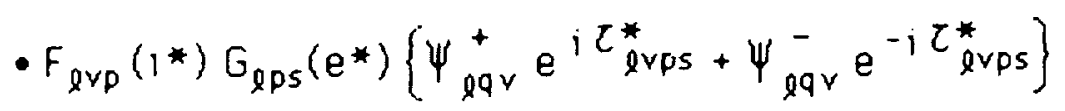

or

$$
\begin{aligned}
& U_{l q, f}^{ \pm}=\Lambda_{l q, f}^{ \pm} \frac{1}{\{l-q) !} \sum_{V, p, s,\{ \pm\}}\left(\frac{R}{q^{*}}\right)^{l+1} F_{l v p}(i *) G_{l p s}\left(e^{*}\right) \\
& \text { - } \psi_{2 q \vee}^{\{ \pm\}} e^{r\left(\{ \pm\} \tau_{g \vee p s}^{*}-q \theta_{g}( \pm) \vartheta+(q-v) \frac{\pi}{2}\right)}
\end{aligned}
$$

This is the desired form of the potential, with both the disturbing body and perturbations referred to the ecliptic. Note that the $\wedge \pm$ qf f still refers to the usual ocean tide coefficients 
The angular argument in equation (213) is given by

$$
\Gamma=\{ \pm\} \tau_{\text {qvps }}^{*}-q \theta_{8}( \pm) g+(q-v) \frac{\pi}{2}
$$

or expanding,

$$
\begin{aligned}
& \Gamma=\{ \pm\}\left[(l-2 p) \omega^{*}+(l-2 p+s) M^{*}+v \Omega^{*}-\frac{\pi}{2} \bmod (l-v, 2)\right]-q \theta_{8}+(q-v) \frac{\pi}{2} \\
& \pm\left[(\mp)\left((2-2 h) \omega^{*}+(2-2 h+j) M^{*}+k \Omega^{*}\right)+m \theta_{8}-\frac{m \pi}{2}+\pi+\varepsilon_{l q, f}^{ \pm}\right]
\end{aligned}
$$

The conditions for secular effects are attained when the time-dependent angular voriables in (2 15) drop out of the equation These conditions are

$$
\begin{aligned}
& \text { 1) }-q \theta_{8} \pm m \theta_{8}=0 ; m, a \geq 0 \\
& \Longrightarrow a=m, \pm 15+ \\
& \text { 2) }\{ \pm\} \vee \Omega^{*} \pm(\mp) k \Omega^{*}=0 ; v, k \geq 0 \\
& \Longrightarrow v=k ;\{ \pm\}=( \pm) \\
& \text { 3) }\{ \pm\}(l-2 p) \pm(F)(2-2 h)=0 \\
& \Longrightarrow l=2, p=n \\
& \text { 4) }\{ \pm\}(l-2 p+s) \pm(F)(2-2 h+j)=0 \Longrightarrow s=\}
\end{aligned}
$$

Note that when $m=0$ or $k=0$, both sign alternatives exist, and we have applied the selection rule appropriate to the general case These degeneracies or symetries are the same as those discussed in Appendix 1 for (1.24) through (1.27).

The onily free variables for the secular effects are $( \pm), h, j, k, m$ 
which are also tre variates which define the cloodsan argument in 1 2 23 Ths implies that each coodson frequency has a secular effect on the ortit af the suri or the moon due ta the + tides. Any secular effect from the - tides are strictly through the $m=0$ or $k=0$ degenerscies nated above, which, in the Doodson develapment. are incoraorated into the uoodsori coefficient amplitudes

The constant phase angle rsec associated with these secular efiects is

$$
\Gamma_{E}=(\pi) \frac{\pi}{2} \operatorname{mog}(2-k, 2)-\frac{k \pi}{2}+\pi+\varepsilon_{2 m, t}^{+}
$$

which ior

$$
\begin{aligned}
k=0 & \Longrightarrow \Gamma_{s e c}=\pi+\varepsilon_{2 m, t}^{+} \\
r=1 \text { for }(+) & \Longrightarrow \Gamma_{\text {sec }}=\varepsilon_{2 m, t}^{+} \\
\operatorname{for}(-) & \Longrightarrow \Gamma_{\text {sec }}=\pi+\varepsilon_{2 m, f}^{+} \\
k=2 & \Longrightarrow \Gamma_{\text {sec }}=\varepsilon_{2 m, j}^{+}
\end{aligned}
$$

The $\pi$ merely indicates a sign change and therefore the $\Gamma_{\mathrm{sec}}$ is nothing other than measure of the phase lag $\varepsilon_{2 m}^{+}, f$ of the ocean tide potentiol.

we can also rewrite the amplitude of the potential in equation (2.13) as a function of the Doodson coefficient, $\bar{A}$, using equation (1.7) of Appendix 1

$$
U_{2 m, f}^{+}=\left\{\frac{\mu_{m}}{\mu_{s}}\left(\frac{\theta_{s}}{\theta_{m}}\right)^{3} \int_{\text {sun }}^{\text {moon }}\left(\frac{R}{g^{*}}\right)^{3} \bar{A}_{f} \wedge_{2 m}^{+} \frac{(-1)^{d}}{\left(2-\delta_{o m}\right)}\left(\frac{3-m}{4}\right) \frac{(2+m) !}{(2-m) !} e^{j \Gamma_{s e c}}\right.
$$


The appropriate real form of the disturbing potential for the secular effects is the real part of the complex potential in (2.19) subject to the conditions of (2.15). Also, as equation (1.8) gives

$$
d=\delta_{o k}+\frac{1}{2}[-k\{\underline{t} k]
$$

it can easily be shown that for any $k$,

$$
(-1)^{d} \cos \Gamma_{\sec }=\cos \varepsilon_{2 m, f}^{+}
$$

Therefore the secular potential is expressed as

$$
U_{2 m, f}^{+}=D_{2 m, f}^{+} \cos \varepsilon_{2 m, f}^{+}
$$

where

$$
D_{2 m, f}^{+}=\left\{\frac{\mu_{m}}{\mu_{s}}\left(\frac{\theta_{s}}{\theta_{m}}\right)^{3}\right\}_{\text {sun }}^{\text {moon }}\left(\frac{R}{a^{*}}\right)^{3} \bar{A}_{f} \wedge_{2 m}^{+} \frac{1}{\left(2-\delta_{o m}\right)}\left(\frac{3-m}{4}\right) \frac{(2+m) !}{(2-m) !}
$$

Of particular interest are the secular changes in the semi-major axis, the eccentricity and the inclination of the orbits of the moon and sun. These secular changes describe the evolution of the orbits of the sun and the moon and furthermore define the secular deceleration in the rotation of the earth, through the balance of the angular momentum equation 
Given a potential $U$, the secular rates in the Kepler elements of the sun and the moon can be computed using the Lagrange Planetary Equations from Kaula (1966, equation 338)

$$
\begin{aligned}
& \frac{d \theta^{*}}{d t}=(\mp) \frac{2}{n^{*} \theta^{*}}(2-2 h+j) D_{2 m, f}^{+} \sin \varepsilon_{2 m, f}^{+} \\
& \frac{d e^{*}}{d t}=(\mp) \frac{\left(1-e^{*}\right)^{\frac{1}{2}}}{n^{*} \theta^{*} e^{*}}\left(\left(1-e^{* 2}\right)^{\frac{1}{2}}(2-2 h+j)-(2-2 h)\right] D_{2 m, f}^{+} \sin \varepsilon_{2 m, f}^{+} \\
& \frac{d^{*}}{d t}=(\mp) \frac{\left.[(2-2 n) \cos )^{*}-k\right]}{n^{*} \theta^{* 2}\left(1-e^{* 2}\right)^{\frac{1}{2}} \sin i^{*}} D_{2 m, f}^{+} \sin \varepsilon_{2 m, f}^{+}
\end{aligned}
$$

where $n^{*}$ is derived from Kepler's law:

$$
n * 2 \theta^{* 3}=\mu+\mu *
$$

When it is desirable to use the rate of change of the mean motion, $\frac{d n^{*}}{d t}$, in place of $\frac{d a^{*}}{d t}$, the appropriate relationship is

$$
\frac{d r_{1}^{*}}{d t}=\frac{-3}{2} \frac{n^{*}}{\theta^{*}} \frac{d \theta^{*}}{d t}
$$

The secular perturbations due to the solid tide are easily computed from equations (2.21) through (2.23) if the correspondences of (169) are applied 



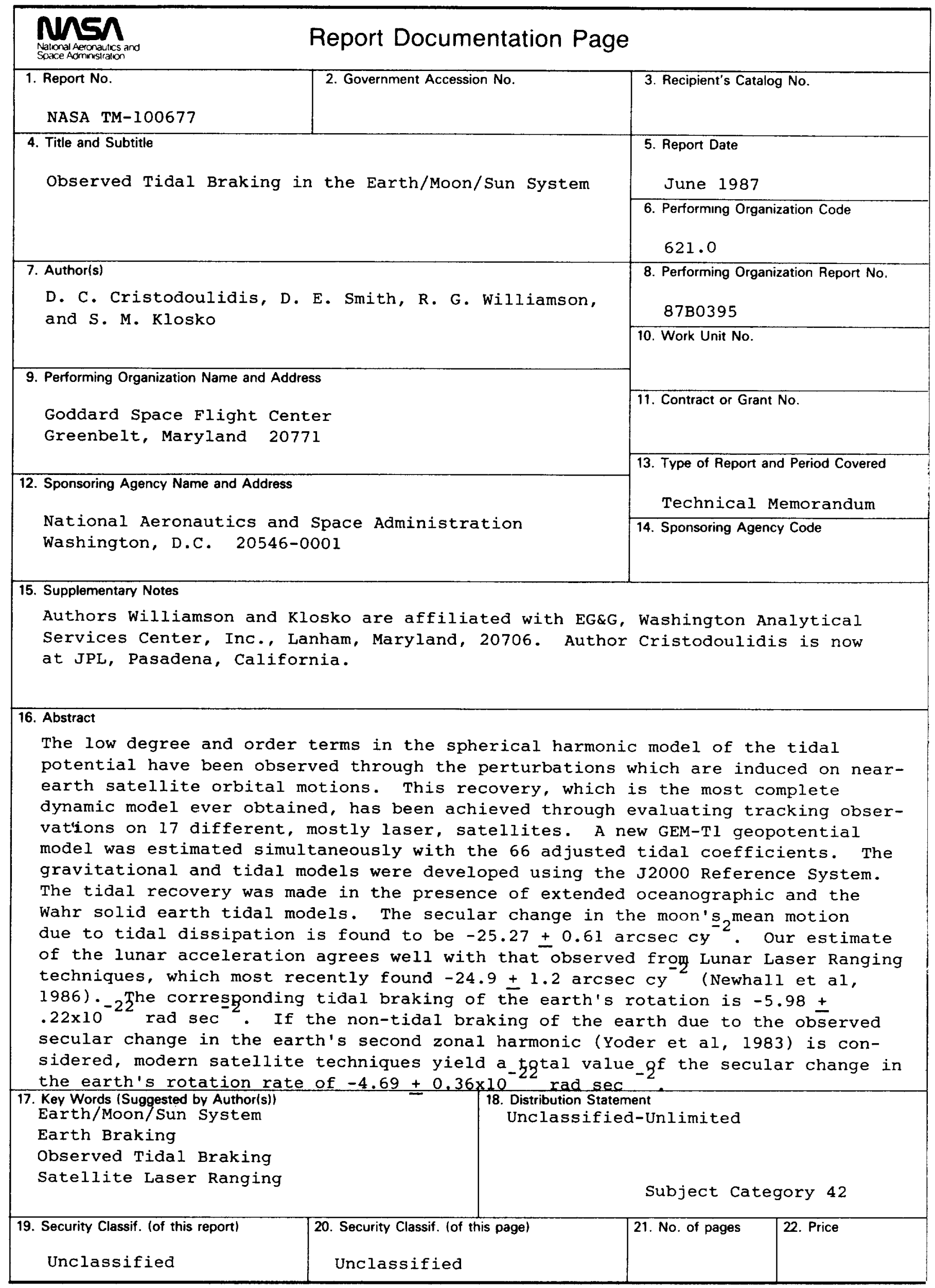


.

.

. 